\title{
Prefrontal cortical activation measured by fNIRS during walking: effects of age, disease and secondary task
}

\author{
Paulo H S Pelicioni ${ }^{1,2}$, Mylou Tijsma $^{3}$, Stephen R Lord ${ }^{1,2}$, Jasmine Menant ${ }^{\text {Corresp. 1,2 }}$ \\ 1 Falls, Balance and Injury Research Centre, Neuroscience Research Australia, Sydney, NSW, Australia \\ 2 School of Public Health and Community Medicine, University of New South Wales, Sydney, NSW, Australia \\ 3 Catharina Hospital, Eindhoven, Netherlands \\ Corresponding Author: Jasmine Menant \\ Email address: j.menant@neura.edu.au
}

Background. Cognitive processes are required during walking to appropriately respond to environmental and task demands. There are now many studies that have used functional Near-Infrared Spectroscopy (fNIRS) to record brain activation to investigate neural bases of cognitive contributions in gait. The aim of this systematic review was to summarize the published research regarding Prefrontal cortical (PFC) activation patterns during simple and complex walking tasks in young adults, older adults and clinical groups with balance disorders using fNIRS. Our secondary aim was to evaluate each included study based on methodological reporting criteria important for good data quality. Methods. We conducted searches in June 2018 using four databases: Embase, PubMed, Scopus and PsycINFO. The strategy search used was: ((((near infrared spectroscopy) OR functional near infrared spectroscopy) OR nirs) OR fnirs) AND (((gait) OR walking) OR locomotion) AND (((((young) OR adult) OR older) OR elderly) NOT children)) AND (((Brain) OR cortex) OR cortical) for our search. The papers included met the specific review criteria: (i) used fNIRS to measure PFC activation patterns; (ii) included walking tasks (simple and complex) and; (iii) assessed young people, older people and/or clinical groups with balance disorders. Results. Thirty five (describing 75 brain activation comparisons) of the 308 studies retrieved through our search met the inclusion criteria. Based on 6 methodological reporting considerations, 20 were of high quality, 10 were of medium quality and 5 were of low quality. Eleven/20 comparisons in young people, 23/37 comparisons in older people and 15/18 comparisons in clinical groups reported increased PFC activation with increased walking task complexity. The majority of comparisons that used verbal fluency, counting backwards or secondary motor tasks reported increases in PFC activation (83\%, 64\% and 58\% of these studies, respectively). In contrast, no studies found secondary visual tasks increased PFC activation. Conclusion. Increased PFC activation was most common in studies that involved walks comprising secondary verbal fluency and arithmetic tasks. Clinical groups generally showed increased PFC activation irrespective of type of secondary task performed during walking which suggests these groups require more attentional resources for safe walking. Systematic review registration number: PROSPERO 2017 - CRD42017059501. 


\section{Prefrontal cortical activation measured by fNIRS}

2 during walking: effects of age, disease and secondary

3 tasks

4

5

Paulo Henrique Silva Pelicioni ${ }^{1,2}$, Mylou Tijsma ${ }^{2}$, Stephen Ronald Lord ${ }^{1,2}$, Jasmine Menant ${ }^{1,2}$

${ }^{1}$ Neuroscience Research Australia, University of New South Wales, New South Wales, Australia

$8 \quad{ }^{2}$ School of Public Health and Community and Medicine, University of New South Wales, New

9 South Wales, Australia

$10{ }^{3}$ Catharina Hospital, Eindhoven, Netherlands

11

12 Corresponding Author:

13 Jasmine Menant ${ }^{1,2}$

14 Neuroscience Research Australia, Margaret Ainsworth Building, 139 Barker Street, Sydney,

15 NSW, 2031, Australia

16 Email address: j.menant@neura.edu.au

17

18 
19

20

21

22

23

24

25

26

27

28

29

30

31

32

33

34

35

36

37

38

39

40

41

42

43

44

45

46

47

48

49

50

51

52

53

54

55

56

57

58

\section{Abstract}

Background. Cognitive processes are required during walking to appropriately respond to environmental and task demands. There are now many studies that have used functional NearInfrared Spectroscopy (fNIRS) to record brain activation to investigate neural bases of cognitive contributions in gait. The aim of this systematic review was to summarize the published research regarding Prefrontal cortical (PFC) activation patterns during simple and complex walking tasks in young adults, older adults and clinical groups with balance disorders using fNIRS. Our secondary aim was to evaluate each included study based on methodological reporting criteria important for good data quality.

Methods. We conducted searches in June 2018 using four databases: Embase, PubMed, Scopus and PsycINFO. The strategy search used was: (((((near infrared spectroscopy) OR functional near infrared spectroscopy) OR nirs) OR fnirs) AND (((gait) OR walking) OR locomotion) AND (((((young) OR adult) OR older) OR elderly) NOT children)) AND (((Brain) OR cortex) OR cortical) for our search. The papers included met the specific review criteria: (i) used fNIRS to measure PFC activation patterns; (ii) included walking tasks (simple and complex) and; (iii) assessed young people, older people and/or clinical groups with balance disorders.

Results. Thirty five (describing 75 brain activation comparisons) of the 308 studies retrieved through our search met the inclusion criteria. Based on 6 methodological reporting considerations, 20 were of high quality, 10 were of medium quality and 5 were of low quality. Eleven/20 comparisons in young people, 23/37 comparisons in older people and 15/18 comparisons in clinical groups reported increased PFC activation with increased walking task complexity. The majority of comparisons that used verbal fluency, counting backwards or secondary motor tasks reported increases in PFC activation $(83 \%, 64 \%$ and $58 \%$ of these studies, respectively). In contrast, no studies found secondary visual tasks increased PFC activation.

Conclusion. Increased PFC activation was most common in studies that involved walks comprising secondary verbal fluency and arithmetic tasks. Clinical groups generally showed increased PFC activation irrespective of type of secondary task performed during walking which suggests these groups require more attentional resources for safe walking.

Systematic review registration number: PROSPERO 2017 - CRD42017059501.

\section{Introduction}

Walking relies heavily upon coordinated movement controlled by subcortical structures such as the Basal Ganglia (Takakusaki, Tomita and Yano, 2008). However, cognition is also important for locomotor tasks, particularly tasks that require attention and processing speed, such as multitasking and gait adaptability (Montero-Odasso et al., 2012; Caetano et al., 2017). Traditionally, the role of cognition has been assessed using dual-task paradigms (walking while performing a secondary cognitive task) which provide indications of the role of attention and executive function in the regulation of gait control (Montero-Odasso et al., 2012) and the negotiation of obstacles (Caetano et al., 2017, 2018). Impaired cognitive processing has been associated with 
59 reduced gait speed and increased gait variability during complex gait (Killane et al., 2014;

60 Hausdorff, 2005), however how higher level brain areas are activated during complex walking

61 tasks is still unclear.

62 Functional near-infrared spectroscopy (fNIRS) is an optical neuroimaging technique for

63 investigating cortical brain area activation while participants move freely. This technique is

64 particularly useful for monitoring hemodynamic responses to brain activation (i.e. changes in

65 oxygenated (oxyHB) and deoxygenated hemoglobin (deoxyHB)) in cortical regions before and

66 after stimulation (i.e. resting followed by simple walking or simple walking followed by dual-

67 task walking) (Leff et al., 2011).

68 Two overlapping theories have been posited for relative changes in cortical activity as measured

69

70

71

72

73

74

75

76

77

78

79

80

81

82

83

84

85

86

87

88

89

90

91

92

93

94

95

96

97

98 with fNIRS. The first suggests reduced activity represents decreased use of a brain region and therefore increased efficiency (Lustig et al., 2009; Grady, 2012). The second suggests increased cortical activity is a compensatory mechanism and reflects over-recruitment and reduced efficiency (Cabeza et al., 2002; Reuter-Lorenz and Cappel, 2008; Grady, 2012).

Several reports of brain activation during walking using fNIRS have been published in the last decade. Activation of the Prefrontal Cortex (PFC) (easily accessible using fNIRS) has often been investigated during walking tasks (Leff et al., 2011). Brain motor areas investigated also include the Pre Motor Cortex (PMC), the Pre Supplementary Motor Area (preSMA), the Supplementary Motor Area (SMA) and the Sensory Motor Cortex (SMC) (Harada et al., 2009; Koenraadt et al., 2014; Lu et al., 2015; Suzuki et al., 2004, 2008). Thus, there is now considerable literature that requires synthesizing and systematic review of the main findings related to the brain activation as assessed by fNIRS during walking tasks.

Some recent reviews have examined fNIRS and gait. These reviews have addressed (i) methodological aspects (Herold et al., 2017; Vitório et al., 2017); (ii) data processing techniques (Vitório et al., 2017); (iii) or restricted their focus to ageing (Vitório et al., 2017; Stuart et al., 2018), Parkinson's disease (PD) or Parkinsonism syndromes (Vitório et al., 2017; Gramigna et al., 2017; Stuart et al., 2018) or Stroke (Gramigna et al., 2017).

Further analysis and synthesis of published fNIRS studies are required to gain a better understanding of (i) brain activation changes during complex walking compared to simple walking or standing; (ii) brain activation patterns in young healthy people as this group provides the model of intact cognitive functioning; and (iii) brain functioning in diverse clinical groups with walking and neurological impairments. A methodological scale is also required to assist in the evaluation of the literature published to date.

Thus, we conducted a systematic review to summarize the published findings regarding brain activation patterns during simple and complex walking tasks in young adults, older adults and clinical groups with balance disorders, to gain an insight into neural processes required for ambulation. Our primary objectives were to determine whether (i) PFC activation patterns change when people perform gait tasks of increasing complexity requiring concomitant somatosensory, motor or cognitive tasks; (ii) PFC activation patterns during gait differ between young and older people and between patient groups and healthy controls. Our secondary aim was 
99 to evaluate each included study based on six methodological reporting criteria important for

100 good data quality.

101

102

103

\section{Methodology}

104

\section{Search strategy}

105

106

107

108

109

110

111

112

113

114

115

116

117

118

119

120

121

122

123

124

125

126

127

128

129

130

131

132

133

134

135

136

137

138

We followed the Preferred Reporting Items for Systematic Reviews and Meta-Analyses (PRISMA) statements and those defined by Moher et al (2009) to identify and screen the articles included in this systematic review. We conducted searches in June 2018 using four databases: Embase, PubMed, Scopus and PsycINFO. A protocol was prospectively registered with the International Prospective Register of Systematic Reviews (PROSPERO) (registration number: PROSPERO 2017: CRD42017059501). We used the following Booleans terms: (((((near infrared spectroscopy) OR functional near infrared spectroscopy) OR nirs) OR fnirs) AND (((gait) OR walking) OR locomotion) AND (((((young) OR adult) OR older) OR elderly) NOT children)) AND (((Brain) OR cortex) OR cortical) for our search. We considered papers in English, Portuguese, Dutch and French.

\section{Selection criteria}

Study identification and screening were conducted independently by PP and MT or PP and JM with disagreements resolved by consultation and input from a third researcher (JM or SL). At stage 1 (identification), the researchers screened the manuscript titles and selected those that were consistent with the broad inclusion criteria. Studies were excluded if: (i) they were not in line with the review objectives; (ii) were conference abstracts with insufficient information for data extraction; (iii) were conducted in animals; (iv) were conducted in children/infants; (v) used fNIRS for other purposes (e.g. muscle studies); (vi) used fNIRS for standalone purposes (i.e. no walking assessment); (vii) used a device other than fNIRS (e.g. electroencephalogram) and/or; (viii) were published in a language other than English, Portuguese, Dutch or French.

At stage 2 (screening), the researchers screened the abstracts to identify papers that met the other specific review criteria: (i) used fNIRS to measure PFC cortical activation patterns; (ii) included walking tasks (simple and complex) other than stepping and; (iii) assessed young people, older people and/or clinical groups with balance disorders (defined as any peripheral or neurological condition that affects balance control). At stage 3 (eligibility), the full-text articles were assessed for eligibility. A manual search for additional relevant references from published reviews and articles was also conducted at this point. Articles were further excluded if: (i) they did not include a walking analysis; (ii) gait tasks involved walking speeds slower than $3 \mathrm{~km} / \mathrm{h}$; (iii) the participants performed tasks other than walking (i.e. stepping tasks); (iv) there was no baseline data comparison. Papers meeting all selection criteria were included at stage 4 (included papers) and relevant information was extracted from the papers by three authors (PP, MT and JM). The primary outcome of the review was PFC activity change post stimulation. This was operationalized by changes in oxyHb, deoxyHb (gold standard measurement in brain magnetic resonance imaging (Obrig and Villringer, 2003; Lindauer et al., 2010)), tissue oxygenation index

Peer) reviewing PDF | (2018:11:32820:1:0:NEW 6 Mar 2019) 
139 (ratio of oxygenated to total tissue hemoglobin) and total hemoglobin level (sum of both oxyHb

140 and deoxyHb). All hemodynamic changes reported in this review reflect statistically significant

141 results reported by the authors of each study, i.e. $p$ values $<0.5$.

142

143 Data extraction

144 From the included studies, relevant data were extracted and summarized for further analysis

145 (Table 1). These included: (i) author and year; (ii) sample characteristics; (iii) study aims; (iv)

146 gait assessment; (v) secondary task types; (vi) equipment details; (vii) fNIRS parameters used to

147 describe the brain activation; (viii) control of motion artefacts and filtering; (ix) main findings;

148 (x) study limitations; and (xi) conclusions.

149 
150

151

152

153

154

155

156

157

158

159

160

161

162

163

164

165

166

167

168

169

170

171

172

173

174

175

176

177

178

179

180

181

182

183

184

185

186

187

188

\section{Table 1: Summary of fNIRS studies.}

PFC: Prefrontal cortex; DT: dual-task; OxyHb: oxygenated hemoglobin; DeoxyHb:

deoxygenated hemoglobin; fNIRS: functional near-infrared spectroscopy; TOI: tissue oxygenated index; MS: Multiple Sclerosis; HOA: healthy older adults; MCI: mild cognitive impairment; PMC: premotor cortex; SMA: supplementary motor area; SMC: sensorimotor cortex; S1: primary sensorimotor cortex; M1: primary motor cortex; Hb: hemoglobin; BA: Brodmann area; PD: Parkinson's disease.

A methodological reporting scale based on availability of information provided in the papers was devised. It comprised one point for the following 6 items: (i) equipment details described adequately, i.e. number of channels for the fNIRS, optode distances; (ii) movement artefacts/high frequency noise controlled for; (iii) use of either the 10-5, 10-10 or 10-20 electroencephalography electrode system to guide the optode placement; (iv) interference with external light controlled for; (v) heart rate changes and physiological noises controlled for; (vi) sample size in each group $>10$. Papers were classified as follows: low methodological reporting quality: total score $\leq 2$ points; medium methodological reporting quality: total score 3 and 4 points; high methodological reporting quality: total score $\geq 5$ points.

\section{Results}

A total of 308 study records were identified from the four databases; 103 unique studies with the removal of duplicates. The manual search of the references of these studies identified 12 further relevant studies. Of these 115 studies, 58 were deemed eligible for full-text assessment based on abstract review, with 35 meeting our final inclusion criteria (Figure 1). The data extracted from these studies are summarized in the Table 1 . Nine studies involved young adults only (simple walking (1), fast walking (1), motor task (2), motor and cognitive tasks (2) and only cognitive tasks (3)); 4 studies involved young and older people (motor and cognitive tasks (1), only cognitive tasks (3)); 1 study involved young and older people and a clinical group with balance disorders (stroke survivors) performing simple walking, motor and cognitive tasks; 10 studies involved older people only (fast walking (2), motor and cognitive tasks (1), somatosensory and cognitive tasks (1), somatosensory and motor and cognitive tasks (1), only cognitive tasks (5)); 8 studies involved older people and clinical groups with balance disorders (simple walking (1), motor and cognitive tasks (2), somatosensory and cognitive tasks (1), only cognitive tasks (4)); 3 studies involved solely clinical groups with balance disorders (simple walking (1) and only cognitive tasks (2)). These studies are summarized in Table 2.

Thirteen studies (Beurskens et al., 2014; Chen et al., 2017; Clark et al., 2014a; Clark et al., 2014b; Hawkins et al., 2018; Lin and Lin, 2016; Lu et al., 2015; Maidan et al., 2016; Mirelman et al., 2014; Mirelman et al., 2017; Nieuwholf et al., 2016; Osofundiya et al., 2016) reported two or more comparisons, i.e. walking while crossing an obstacle and walking while performing a serial subtraction, both in comparison to a baseline condition such as simple walking. Many 
189

190

191

192

193

194

195

196

197

198

199

200

201

202

203

204

205

206

207

208

209

210

211

212

213

214

215

216

217

218

219

220

221

222

223

224

225

226

227

228

studies also contrasted brain activation in more tha one area. In total, 75 brain activation comparisons are included in this review.

\section{Figure 1: Flowchart of the articles selection process.}

Table 2: Effect of the additional tasks relative to baseline conditions on regional cortical activation in healthy young adults, healthy older adults and clinical groups with balance disorders.

PFC: Prefrontal cortex; preSMA: Pre-supplementary motor area; SMA: Supplementary motor area; S1: Primary sensorimotor cortex; M1: Primary motor cortex; PMC: Premotor cortex; mSMC: Medial sensorimotor cortex; $(+)$ higher activation when performing the additional task; (-) lower activation when performing the additional task; $(=)$ no changes in activation; Baseline comparison: ${ }^{1}$ standing still; ${ }^{2}$ simple walking; ${ }^{3}$ easier level of the secondary task. Significant differences only for: ${ }^{4}$ first half of the task; ${ }^{5}$ second half of the task; ${ }^{6}$ obstacle negotiation and wearing a vest with $10 \%$ body weight conditions; ${ }^{7}$ walk vs. visual task; ${ }^{8}$ walk vs alphabet recall; ${ }^{9}$ overground walking; ${ }^{10}$ walking and counting backwards task.

aAtaxia; 'bStroke; 'Parkinson's disease; ${ }^{\mathrm{d} O b e s i t y ;}{ }^{\mathrm{e} M u l t i p l e ~ S c l e r o s i s ;}{ }^{\mathrm{f}}$ Mild Cognitive Impairment; ${ }^{g}$ Neurological gait.

Table 3 presents the individual and overall methodological reporting scores, and also describes how the scores were attributed for each paper. Twenty studies $(57 \%)$ were classified as high quality, 10 (29\%) as medium quality and five (14\%) as low quality. Figure 2 shows the number of comparisons from the included studies showing an increase, decrease or no change in PFC activation when comparing either a) walking to standing or b) walking with an additional task to simple walking, according to group. In young people, 11 of the 20 comparisons from 13 studies reported significant increases in PFC activation (Lu et al., 2015; Suzuki et al., 2004; Lin and Lin, 2016; Hill et al., 2013; Holtzer et al., 2011; Maidan et al., 2018; Mirelman et al., 2017; Meester et al., 2014; Mirelman et al., 2014); five reported a reduction in PFC activation (Hawkins et al., 2018; Koenraadt et al., 2014; Lin and Lin, 2016) and four reported no change (Koenraadt et al., 2014; Beurskens et al., 2014; Hawkins et al., 2018; Takeuchi et al., 2016). Although the aim of the study was to report changes in PFC activation, three studies (four comparisons) have also investigated activation in additional cortical areas in young people (Table 1) (Lu et al., 2015; Koenraadt et al., 2014; Suzuki et al., 2004). Of these, two comparisons indicated increased cortical activation in the SMA (Lu et al., 2015), while one found no change in the SMA as well as no change in brain activation in the preSMA and motor and sensorimotor areas (Primary Motor Cortex (M1) and Primary Sensorimotor Cortex (S1)) (Koenraadt et al., 2014). One comparison also reported increased cortical activation in the PMC ( $\mathrm{Lu}$ et al., 2015) while another found no change in activation in the PMC or the SMC (Suzuki et al., 2004). Of the 37 comparisons from 22 studies conducted in older adults, 23 reported significant increases in PFC activation with increasing locomotor task complexity (Lucas et al., 2018; Mirelman et al., 2017; 
229

230

231

232

233

234

235

236

237

238

239

240

241

242

243

244

245

246

247

248

249

250

251

252

253

254

255

256

257

258

259

260

261

262

263

264

265

266

267

268

269

Hawkins et al., 2018; Chaparro et al., 2017; Chen et al., 2017; Harada et al., 2009; Holtzer et al., 2011, 2015, 2016, 2017a, 2017b, Maidan et al., 2016; Verghese et al., 2017; Clark et al., 2014a, 2014b; Osofundiya et al., 2016; Hernandez et al., 2016); two reported a reduction in PFC activation (Beurskens et al., 2014; Clark et al., 2014a) and twelve reported no change (Hawkins et al., 2018; Eggenberger et al., 2016; Mori et al., 2018; Harada et al., 2009; Takeuchi et al., 2016; Clark et al., 2014b; Maidan et al., 2016; Caliandro et al., 2015; Al-Yahya et al., 2016). Only one study investigated other cortical areas, reporting no change in pre-SMA or Medial Sensorimotor Cortex (mSMC) activation and an increase in SMA activation (Harada et al., 2009). Finally, of the 18 comparisons from twelve studies conducted in clinical groups, fifteen reported increased PFC activation (Thumm et al., 2018; Hawkins et al., 2017; Chaparro et al., 2017; Takeuchi et al., 2016; Osofundiya et al., 2016; Hernandez et al., 2016; Holtzer et al., 2016; Maidan et al., 2016; Caliandro et al., 2015; Al-Yahya et al., 2016; Doi et al., 2013) and three comparisons found no change (Mori et al., 2018; Maidan et al., 2016).

Table 3: Methodological reporting criteria ratings for the included studies.

Figure 2: Proportion of comparisons within each group showing increase (up arrows), decrease (down arrows) or no change (circle) in Prefrontal Cortex (PFC) activation when comparing walking with secondary task versus baseline.

There was no indication that the PFC activation was associated with methodological reporting scores; i.e. increased activation was reported in 23/36 (64\%) comparisons in high quality studies, $14 / 18(78 \%)$ comparisons in medium quality studies and 10/19 (53\%) comparisons in low quality studies; $\left(\chi^{2}=2.56, \mathrm{df}=2, \mathrm{p}=0.279\right)$ (Table 4). Regarding the effects of a secondary task during walking on PFC activation, 9/14 (64\%) comparisons that used counting backwards reported increases, 20/24 (83\%) that used verbal fluency reported increases, 11/19 (58\%) that used complex motor tasks reported increases and $0 / 4(0 \%)$ that used visual tasks reported increases (Table 5).

\section{Table 4: Prefrontal cortical activation in relation to methodological reporting scale.}

Table 5: Prefrontal cortical activation in relation to complex walking tasks.

Table 6 shows the effect of an additional motor or cognitive task relative to simple walking on gait outcomes in healthy young adults, healthy older adults and clinical groups with balance disorders. Reduced gait speed was reported in all studies investigating overground walking with the exception of one study that observed no changes in gait speed when older people walked while counting backwards by 3 or negotiated an obstacle course (Mirelman et al., 2017). No changes in gait speed were also observed in the two studies of treadmill walking where walking speed was controlled by the examiner (Clark et al., 2014a, 2014b). Shorter step/stride length was observed in all studies conducted on level surfaces except for one study that investigated 
270

271

272

273

274

275

276

277

278

279

280

281

282

283

284

285

286

287

288

289

290

291

292

293

294

295

296

297

298

299

300

301

302

303

304

305

306

307

308

309

counting backwards from a 3-digit number while walking on a treadmill (Al-Yahya et al., 2016) where both older people and stroke survivors exhibited increased stride length compared with simple walking. As expected, higher spatiotemporal variability was observed when people performed a secondary motor task that manipulated spatiotemporal characteristics, such as obstacle crossing and precision stepping (Koenraadt et al., 2014; Clark et al., 2014b; Mirelman et al., 2017), but also in one study where older people performed a verbal fluency task (Clark et al., 2014a). No changes in gait variability were observed when young people walked while performing arithmetic tasks (Lu et al., 2015; Meester et al., 2014), when young people walked carrying a tray (Lu et al., 2015), when somatosensory information was manipulated in older people (Clark et al., 2014a, 2014b) and when young and older people walked while negotiating obstacles or while counting backwards by 3 (Mirelman et al., 2017). One study, by Nieuwholf and colleagues (2016), showed decreased stride length variability in people with PD when performing the digit span task while walking.

Table 6: Effect of the additional tasks on gait outcomes compared to simple walking in healthy young adults, healthy older adults and clinical groups with balance disorders. $(+)$ increase of spatiotemporal parameter when performing the additional task during walking; (-) decrease of spatiotemporal parameter when performing the additional task; $(=)$ no changes in spatiotemporal parameter.

Significant differences only for: ${ }^{1}$ obstacle negotiation; ${ }^{2}$ treadmill walking; ${ }^{3}$ overground walking; ${ }^{4}$ walking + subtracting by $7 \mathrm{~s} ;{ }^{5}$ walking while reciting digit spans.

Table 7 presents group comparisons with respect to brain activation changes resulting from undertaking a complex walk between (i) healthy older and young adults and (ii) clinical groups with balance disorders and healthy peers. Of the five studies that contrasted PFC activation changes when conducting a dual task walk between young and older adults, only one study showed greater increases in PFC activation in older adults in all tasks performed (Mirelman et al., 2017). Another study (Hawkins et al., 2018), reported greater PFC activation in older people in simple walking in only the first half of an obstacle negotiation task and not when walking and performing a verbal fluency task. Two other studies reported no group differences (Beurskens et al., 2014; Takeuchi et al., 2016) and one reported a relatively smaller increase in PFC activation in older people (Holtzer et al., 2011).

Table 7: Prefrontal cortical activation pattern differences between healthy young and older adults and between clinical groups with balance disorders and healthy peers.

DT: Dual-task; SW: Simple walking; OBS: Obstacle negotiation; a: first half of the task; b: second half of the task; PB: Partial body support; PS: Precision stepping; $(+)$ higher activation when performing the additional task; (-) lower activation when performing the additional task; $(=)$ no change in activation. 
310 Thirteen comparisons from eight studies have contrasted PFC activation changes when

311 conducting a dual task walk between clinical groups with balance disorders and healthy peers.

312 Four of these reported a relatively larger increase in PFC activation in clinical groups with

313 balance disorders. Two comparisons showed increased PFC activation when people with

314 multiple sclerosis performed cognitive dual tasks (Chaparro et al., 2017; Hernandez et al., 2016),

315 while the other comparisons showed increased PFC activation when stroke survivors walked

316 while performing a cognitive dual task (Al-Yahya et al., 2016) or in the first half of an obstacle

317 negotiation task (Hawkins et al., 2018). Six comparisons reported no between-group PFC

318 activation differences. In three of these comparisons, stroke survivors (Hawkins et al., 2018), PD

319 (Maidan et al., 2016) and obesity (Osofundiya et al., 2016) performed motor tasks (obstacle

320 negotiation and precision stepping), while in three other comparisons, stroke survivors (Hawkins

321 et al., 2018), neurological gait (Holtzer et al., 2016) and obesity (Osofundiya et al., 2016)

322 performed cognitive tasks. Finally, the three reports of PFC decreases in clinical groups were

323 observed when people with multiple sclerosis walked with partial body support (Chaparro et al.,

324 2017) and when people with PD and stroke performed a cognitive dual task (Maidan et al., 2016;

325 Mori et al., 2018).

326

327

328

\section{Discussion}

329

This systematic review summarizes the published findings regarding PFC cortical patterns of activation in healthy young adults, healthy older adults and clinical groups with balance

330 disorders, to gain an insight into neural processes during simple and complex walking tasks. Approximately $60 \%$ of the studies comparisons reported that healthy young and older adults

332 exhibited higher PFC activation when performing a complex task while walking compared with

333 a baseline simple walking task; this was also the case for more than $80 \%$ of studies comparisons of clinical groups with balance disorders. Moreover, PFC activation appears to be related to the type of complex walk undertaken.

336

337

\section{Brain activation in healthy young adults}

338

339 Compared with simple walking, PFC activation increased when individuals performed (i) fast walks (Suzuki et al., 2004); (ii) negotiated expected obstacles (Mirelman et al., 2017; Maidan et al., 2018) and unexpected obstacles of different heights (Maidan et al., 2018a); (iii) a secondary task while walking (Holtzer et al., 2011; Mirelman et al., 2014); the last being the paradigm used in most studies that have assessed brain activation patterns in healthy young adults. In these studies, participants performed the following secondary tasks: subtracting numbers by 1,3 or 7 (Hill et al., 2013; Meester et al., 2014; Mirelman et al., 2014; Mirelman et al., 2017), walking while talking (Holtzer et al., 2011) and talking and carrying a bottle of water on a tray (Lu et al., 2015). However, increased hemodynamic responses in the PFC appear to be task-specific. Indeed, several studies of healthy young adults (Hawkins et al., 2018; Koenraadt et al., 2014; Lin and Lin, 2016; Beurskens et al., 2014; Takeuchi et al., 2016) reported no change or even a decrement in PFC activation during either secondary motor task performance (e.g. precision 
350

351

352

353

354

355

356

357

358

359

360

361

362

363

364

365

366

367

368

369

370

371

372

373

374

375

376

377

378

379

380

381

382

383

384

385

386

387

388

389

stepping, crossing obstacles or walking on a narrow pathway) or cognitive task performance (e.g. visual checking, alphabet recall, memory task and manipulating a smartphone). Such findings suggest that increasing balance/ locomotor changes does not require additional PFC activation in healthy young adults, and might involve other cortical and subcortical areas involved in the control of locomotion. In addition, secondary tasks involving working memory (Lin and Lin, 2016; Beurskens et al., 2014) might also involve cortical areas in addition to the PFC that were not investigated in the published studies.

\section{Brain activation in healthy older adults}

Most studies (Lucas et al., 2018; Mirelman et al., 2017; Hawkins et al., 2018; Chaparro et al., 2017; Chen et al., 2017; Holtzer et al., 2011, 2015, 2016, 2017a, 2017b; Clark et al., 2014a, 2014b; Osofundiya et al., 2016; Hernandez et al., 2016; Maidan et al., 2016; Verghese et al., 2017) but not all (Al-Yahya et al., 2016; Beurskens et al., 2014; Takeuchi et al., 2016; Hawkins et al., 2018; Eggenberger et al., 2016; Harada et al., 2009; Mori et al., 2018;) reported that when healthy older adults performed cognitive or motor tasks while they walked, the PFC was more activated in comparison to baseline conditions. It seems that in healthy older adults, PFC activation increases with secondary cognitive tasks that involve attention and executive functioning (e.g. walking while subtracting). In contrast, and similar to what is noted in healthy young adults, the conduct of tasks that require speed manipulation (Harada et al., 2009; Eggenberger et al., 2016), visual checking (Beurskens et al., 2014), unpractised tasks (manipulating a smartphone) (Takeuchi et al., 2016), or obstacle negotiation (Hawkings et al., 2018; Maidan et al., 2016) do not appear to increase PFC activation. As with young adults, increased activation may occur in other cortical areas that process visual-spatial stimuli (Wu et al., 2018).

\section{Brain activation in healthy individuals - young: older comparisons}

Healthy older adults usually walk slower and have more difficulty performing dual tasks than healthy young adults, (Al-Yahya et al., 2011). However, of the five studies that investigated between age-group effects when performing walks with secondary tasks, only one study observed greater PFC activation in older people performing different secondary tasks (obstacle negotiation and counting backwards) (Mirelman et al., 2017). PFC activation patterns were not different between healthy young and older adults in three studies (Beurskens et al., 2014; Takeuchi et al., 2016; Hawkins et al., 2018) and lower in older adults in one (Holtzer et al., 2011). The limited number of studies which have explored the effects of aging as well as the nature of the secondary task used in these studies might account for the lack of an age effect.

\section{Brain activation in clinical groups with balance disorders}

In most clinical groups with balance disorders, including stroke survivors (Al-Yahya et al., 2016; Hawkins et al., 2018), obese individuals (Osofundiya et al., 2016), individuals with ataxia (Caliandro et al., 2015), multiple sclerosis (Chaparro et al., 2017; Hernandez et a., 2016), 
390

391

392

393

394

395

396

397

398

399

400

401

402

403

404

405

406

407

408

409

410

411

412

413

414

415

416

417

418

419

420

421

422

423

424

425

426

427

428

429

peripheral neuropathy (Holtzer et al., 2016), and mild cognitive impairment (Doi et al., 2013), higher PFC activation has been reported regardless of the type of concomitant task performed during ambulation. This is also the case for comparisons made in studies in people with PD (Maidan et al., 2016, Thumm et al., 2018 Nieuwholf et al., 2016) with one exception; Maidan et al found no change in PFC activation when comparing walking whilst performing a concomitant subtracting task with simple walking (Maidan et al., 2016).

\section{Theoretical considerations}

Our findings of enhanced hemodynamic responses in the PFC apparent when older adults and individuals with balance disorders perform complex walking tasks align particularly with the notion that increased cortical activity reflects a compensatory mechanism (Cabeza et al., 2002; Reuter-Lorenz and Cappel, 2008; Grady, 2012). This might reflect the need to allocate more attentional resources to walking while performing secondary tasks, or the need to use a more direct locomotor pathway due to deficits in automaticity (e.g. as generally observed in individuals with PD) (Herold et al., 2017). The age-related differences are also consistent with the frontal lobe hypothesis of aging (West, 1996) and the cognitive reserve theory which supports that older adults increase brain activity by a larger degree to cope with elevated cognitive task difficulty (Stern, 2009). Moreover, these functional effects of aging mirror agerelated structural changes proposed by the "last-in-first-out" hypothesis where late maturing brain regions decline first in later life (Raz and Kennedy, 2009; Tamnes et al., 2013; Bender, Volkle and Raz, 2015) and explain gait disturbances.

\section{Differential effects of secondary task type on PFC activation}

Four secondary task types were commonly used in the included studies: counting backwards, verbal fluency, motor tasks and visual tasks. Of these, verbal fluency was the most consistent in increasing PFC activation (Lucas et al., 2018; Hawkins et al., 2018; Chaparro et al., 2017; Chen et al., 2017; Holtzer et al., 2011, 2015, 2016, 2017a, 2017b; Clark et al., 2014a, 2014b; Osofundiya et al., 2016; Hernandez et al., 2016; Verghese et al., 2017; Doi et al., 2013). Counting backwards also increased PFC in most studies (Lu et al., 2015; Hill et al., 2013; Meester et al., 2014; Mirelman et al., 2014; Mirelman et al., 2017; Maidan et al., 2016; Al-Yahya et al., 2016; Nieuwhof et al., 2016). Of the 9 comparisons that did not show increased PFC activation with verbal fluency or backward counting tasks, some were from studies of low (Hawkins et al., 2018) or medium methodological reporting quality (Lin and Lin, 2016) that did not report control for motion artefacts, external lighting and physiological noise, and in two studies (Beurskens et al., 2014; Al-Yahya et al., 2016) the secondary tasks were performed on a treadmill. This may be important as Clark et al (2014a) observed that when participants walked on treadmill no change in PFC activation was observed between dual-task (verbal task) and baseline condition, while changes were observed on overground walking.

Half of the studies that examined the effects of motor tasks during walking in healthy individuals found increases in PFC. In the studies where PFC was not increased, three were performed on a 
430 treadmill (Eggenberger et al., 2016; Harada et al., 2009; Koenraadt et al., 2014), one was of low 431 methodological reporting quality (Hawkins et al., 2018), one did not control for important 432 aspects that could have affected the interpretation of the data, such as motion artefacts, external 433 lighting and physiological noise (Lin and Lin, 2016), and one was conducted in people with PD 434 (Maidan et al., 2016). Obstacle negotiation and precision stepping tasks increased PFC in the 435 three studies performed in clinical groups with balance disorders (Osofundiya et al., 2016;

436 Maidan et al., 2016; Hawkins et al., 2018), but this may simply indicate any additional load may 437 illicit such changes in such populations.

438 Finally, visual tasks such as visual checking and manipulating a smartphone did not increase 439 PFC activation (Beurskens et al., 2014; Takeuchi et al., 2016). For these task types, other brain 440 areas such as the visual cortex might be more involved. However, further studies directly 441 assessing visual cortex activation as well as other cortical regions are required to confirm this 442 hypothesis.

443

444 Study limitations

445 Studies addressing gait with fNIRS are still at a relatively early stage, with best practice 446 methodology evolving as experience with this technique is garnered. Studies may have not met particular quality criteria due to the pioneering nature of the studies using this new technology and/or omission of reporting of all methodological factors and most of the papers $(89 \%)$ had one

450

451

452

453

454

455

456

457

458

459

460

461

462

463

464

465

466

467

468

469 or more of the following methodological limitations: small sample sizes, no indication of removal or control of motion artefacts or physiological noise in data processing and sub-optimal number and positioning of optodes. Further, the secondary tasks used in many studies involved speaking (counting backwards and verbal fluency) requiring muscles adjacent to the PFC (Zimeo-Morais et al., 2018). Such muscle activity as well as different facial expressions (Balardin et al., 2017) may affect fNIRS signal quality.

To address the above, we recommend that in future studies, sample sizes be based on power analyses of expected effect sizes for spatiotemporal gait and hemodynamic measures to provide confidence in the study findings. Second, motion artefacts should be removed during the data processing or be controlled for; confounding physiological noise such as fluctuations in heart rate should either be monitored and reported, or controlled for using appropriate filtering. Third, baseline and test trials should be of sufficient duration to detect the slow changing hemodynamic signals as oxygenated blood starts flowing between 1 and 2 s after stimuli onset and achieves its peak approximately 6s after stimulus onset (Holtzer et al., 2011). Finally, EEG standards (i.e. 105) and/or anatomical maps to define optode positions (i.e. Brodmann areas) for the brain regions of interest should be used. However, we acknowledge that given the limited number of fNIRS channels in current devices, this may prove to be an unavoidable limitation for some investigations (Koenraadt et al., 2014; Maidan et al., 2016).

We also acknowledge other limitations. First, there was considerable heterogeneity of study protocols. As such, variations in baseline conditions (e.g. sitting/standing/unspecified), walking speed (e.g. self-selected/controlled), duration and amount of trials, treadmill vs. overground 
470 walking, montage, inter-optode distance, etc. limited the clustering of studies and hindered the 471 overall interpretation of the data. Finally, factors such as motor repertoire, physical activity, 472 practice and skill levels, risk of falling and hemispheric asymmetry (Ekkekakis, 2009; Erickson 473 et al., 2007; Jancke, Shah and Peters, 2000; Naito and Hirose, 2014) can affect cortical activity 474 but were beyond the scope of this review. Complementary studies and reviews are required to 475 elucidate the influence these factors have on cortical activity and associated balance control. 476

\section{Conclusion}

478 This systematic review found the majority of studies found increased PFC activation with 479 increased walking task complexity in young and older people and clinical groups with balance 480 disorders. However, increased PFC activation was most common in studies that contained walks

481

482

483

484

485

486

487

488

489

490

491

492

493

494

495

496

497

498

499

500

501

502

503

504

505

506

507

508 comprising secondary tasks of verbal fluency, arithmetic and alphabet reciting. The finding that clinical groups with balance disorders generally showed increased PFC activation irrespective of type of secondary task during walking suggests these groups require more attentional resources for safe walking.

\section{References}

Al-Yahya, E., Dawes, H., Smith, L., Dennis, A., Howells, K., Cockburn, J., 2011. Cognitive motor interference while walking: a systematic review and meta-analysis. Neurosci. Biobehav. Rev. 35, 715-728.

Al-Yahya, E., Johansen-Berg, H., Kischka, U., Zarei, M., Cockburn, J., Dawes, H., 2016. Prefrontal cortex activation while walking under dual-task conditions in stroke: a multimodal imaging study. Neurorehabil. Neural Repair 30, 591-599.

Balardin, J.B., Zimeo-Morais, G.A., Furucho, R.A., Trambaiolli, L.R., Sato, J.R., 2017. Impact of communicative head movements on the quality of functional near-infrared spectroscopy signals: negligible effects for affirmative and negative gestures and consistent artifacts related to raising eyebrows. J. Biomed. Opt. 22, article 46010.

Bender, A.R., Volkle, M.C., Raz, N., 2015. Differential aging of cerebral white matter in middleaged and older adults: a seven-year follow-up. Neuroimage. 125, 74-83.

Beurskens, R., Helmich, I., Rein, R., Bock, O., 2014. Age-related changes in prefrontal activity during walking in dual-task situations: a fNIRS study. Int. J. Psychophysiol. 92, 122-128.

Cabeza, R., Anderson, N.D., Locantore, J.K., McIntosh, A.R., 2002. Aging gracefully: compensatory brain activity in high-performing older adults. Neuroimage. 17, 1394-1402. 
509 Caetano, M.J.D., Menant, J.C., Schoene, D., Pelicioni, P.H.S., Sturnieks, D.L., Lord, S.R., 2017.

510 Sensorimotor and cognitive predictors of impaired gait adaptability in older people. J. Gerontol.

511 A Biol. Sci. Med. Sci. 72, 1257-1263.

512

513 Caetano, M.J.D., Lord, S.R., Brodie, M.A., Schoene, D., Pelicioni, P.H.S., Sturnieks, D.L.,

514 Menant, J.C., 2018. Executive functioning, concern about falling and quadriceps strength

515 mediate the relationship between impaired gait adaptability and fall risk in older people. Gait

516 Posture 59, 188-192.

517

518 Caliandro, P., Serrao, M., Padua, L., Silvestri, G., Iacovelli, C., Simbolotti, C., Mari, S., Reale,

519 G., Casali, C., Rossini, P.M., 2015. Prefrontal cortex as a compensatory network in ataxic gait: a

520 correlation study between cortical activity and gait parameters. Restor. Neurol. Neurosci. 33,

521 177-187.

522

523 Chaparro, G., Balto, J.M., Sandroff, B.M., Holtzer, R., Izzetoglu, M., Motl, R.W., Hernandez,

524 M.E., 2017. Frontal brain activation changes due to dual-tasking under partial body weight

525 support conditions in older adults with multiple sclerosis. J. Neuroeng. Rehabil. 14, 65.

526

527 Chen, M., Pillemer, S., England, S., Izzetoglu, M., Mahoney, J.R., Holtzer, R., 2017. Neural

528 correlates of obstacle negotiation in older adults: an fNIRS study. Gait Posture 58, 130-135.

529

530 Clark, D.J., Christou, E.A., Ring, S.A., Williamson, J.B., Doty, L., 2014a. Enhanced

531 somatosensory feedback reduces prefrontal cortical activity during walking in older adults. J.

532 Gerontol. A Biol. Sci. Med. Sci. 69, 1422-1428.

533

534 Clark, D.J., Rose, D.K., Ring, S.A., Porges, E.C., 2014b. Utilization of central nervous system

535 resources for preparation and performance of complex walking tasks in older adults. Front.

536 Aging Neurosci. 25, 217.

537

538 Doi, T., Makizako, H., Shimada, H., Park, H., Tsutsumimoto, Uemura, K., Suzuki, T., 2013.

539 Brain activation during dual-task walking and executive function among older adults with mild

540 cognitive impairment: a fNIRS study. Aging Clin. Exp. Res. 25, 539-544.

541

542 Eggenberger, P., Wolf, M., Schumann, M., de Bruin, E.D., 2016. Exergame and balance training

543 modulate Prefrontal Brain activity during walking and enhance executive function in older

544 adults. Front Aging Neurosci. 8, article 66.

545

546 Ekkekakis, P., 2009. Illuminating the black box : investigating prefrontal cortical hemodynamics

547 during exercise with near-infrared spectroscopy. J. Sport Exerc. Psychol. 31, 505-553. 
549 Erickson, K.I., Colcombe, S.J., Wadhwa, R., Bherer, L., Peterson, M.S., Scalf, P.E., Kim, J.S.,

550 Alvarado, M., Kramer, A.F., 2007. Training-induced plasticity in older adults : effects of training

551 on hemispheric asymmetry. Neurobiol. Aging 28, 272-283.

552

553 Grady, C., 2012. The cognitive neuroscience of aging. Nat. Rev. Neurosci. 13, 491-505.

554

555 Gramigna, V., Pellegrino, G., Cerasa, A., Cutini, S., Vasta, R., Olivadese, G., Martino, I.,

556 Quattrone, A., 2017. Near-infrared spectroscopy in gait disorders: is it time to begin?

557 Neurorehabil. Neural Repair 31, 402-412.

558

559

Harada, T., Miyai, I., Suzuki, M., Kubota, K., 2009. Gait capacity affects cortical activation

560 patterns related to speed control in the elderly. Exp. Brain Res. 193, 445-454.

561

562

Hausdorff, J.M., 2005. Gait variability: methods, modelling and meaning. J. Neuroeng. Rehabil. $5632,19$.

564

565

Hawkins, K.A., Fox, E.J., Daly, J.J., Rose, D.K., Christou, E.A., McGuirk, T.E., Otzel, D.M.,

566 Butera, K.A., Chatterjee, S.A., Clark, D.J., 2018. Prefrontal over-activation during walking in

567 people with mobility deficits: interpretation and functional implications. Hum. Mov. Sci. 59, 4656855.

569

570

Hernandez, M.E., Holtzer, R., Chaparro, G., Jean, K., Balto, J.M., Sandroff, B.M., Izzetoglu, M., Moti, R.W., 2016. Brain activation changes during locomotion in middle-aged to older adults

572 with multiple sclerosis. J. Neurol. Sci. 15, 277-283.

573

574

Herold, F., Wiegel, P., Scholkmann, F., Thiers, A., Hamacher, D., Schega, L., 2017. Functional

575 near-infrared spectroscopy in movement science: a systematic review on cortical activity in

576

577

578 postural and walking tasks. Neurophotonics 4, 041403.

579

Hill, A., Bohil, C., Lewis, J., Neider, K., 2013. Prefrontal cortex activity during walking while 580 multitasking: an fNIR study. Proc. Hum. Factors Ergon. Soc. Annu. Meet. 57, 1224-1228.

Holtzer, R., Mahoney, J.R., Izzetoglu, M., Izzetoglu, K., Onaral, B., Verghese, J., 2011. fNIRS Sci. Med. Sci. 66, 879-887.

584 
589 Holtzer, R., Verghese, J., Allali, G., Izzetoglu, M., Wang, C., Mahoney, J.R., 2016. Neurological 590 gait abnormalities moderate the functional brain signature of the posture first hypothesis. Brain

591 Topogr. 29, 334-343.

592

593 Holtzer, R., Schoen, C., Demetriou, E., Mahoney, J.R., Izzetoglu, M., Wang, C., Verghese, J., 594 2017a. Stress and gender effects on prefrontal cortex oxygenation levels assessed during single 595 and dual-task walking conditions. Eur. J. Neurosci. 45, 660-670.

596

597

Holtzer., R., Yuan, J., Verghese, J., Mahoney, J.R., Izzetoglu, M., Wang, C., 2017b. Interactions

598 of subjective and objective measures of fatigue defined in the context of brain control of

599 locomotion. J. Gerontol. A Biol. Sci. Med. Sci. 72, 417-423.

600

601

Jancke, L., Shah, N.J., Peters, M., 2000. Cortical activation in primary and secondary motor

602 areas for complex bimanual movements in professional pianists. Brain Res. Cogn. Brain Res. 10,

603 177-183.

604

605

Killane, I., Donoqhue, O.A., Savva, G.M., Cronin, H., Kenny, R.A., Reilly, R.B., 2014. Relative 606 association of processing speed, short-term memory and sustained attention with task on gait

607

608 speed: a study of community-dwelling people 50 years and older. J. Gerontol. A Biol. Sci. Med.

609

610 Sci. 69, 1407-1414.

611

Koenraadt, K.L., Roelofsen, E.G., Duysen, J., Keijsers, N.L., 2014. Cortical control of normal

612

613 gait and precision stepping: an fNIRS study. Neuroimage 85, 415-422.

614

Leff, D.R., Orihuela-Espina, F., Elwell, C.E., Athanasiou, T., Delpy, D.T., Darzi, A.W., Yang,

615 G.Z., 2011. Assessment of the cerebral cortex during motor task behaviours in adults: a

616 systematic review of functional near infrared spectroscopy (fNIRS) studies. Neuroimage 54,

617

618 2922-2936.

619

620

621

Lin, M.I., Lin, K.H., 2016. Walking while performing working memory tasks changes the prefrontal cortex hemodynamic activations and gait kinematics. Front. Behav. Neurosci. 10, 92.

622

Lindauer, U., Dirnagl, U., Fuchtemeier, M., Bottiger, C., Offenhauser, N., Leithner, C., Royl, G.,

623 2010. Pathophysiological interference with neurovascular coupling - when imaging based on haemoglobin might go blind. Front. Neuroenergetics 2, article 25.

624

625

Lu, C.F., Liu, Y.C., Yang, Y.R., Wu, Y.T., Wang, R.Y., 2015. Maintaining gait performance by

626 cortical activation during dual-task interference: a functional near-infrared spectroscopy study.

627 PLoS One 10, e0129390.

628 
629 Lucas, M., Wagshul, M.E., Izzetoglu, M., Holtzer, R., 2018. Moderating effect of white matter 630 integrity on brain activation during dual-task walking in older adults. J. Gerontol. A Biol. Sci.

631 Med. Sci. accepted.

632

633 Lustig, C., Shah, P., Seidler, R., Reuter-Lorenz, P.A., 2009. Aging, training, and the brain: a 634 review and future directions. Neuropsychol. Rev. 19, 504-522.

635

636 Maidan, I., Nieuwhof, F., Bernad-Elazari, H., Reelick, M.F., Bloem, B.R., Giladi, N., Deutsch, 637 J.E., Hausdorff, J.M., Claassen, J.A., Mirelman, A., 2016. The role of the frontal lobe in complex 638 walking among patients with Parkinson's disease and healthy older adults: an fNIRS study.

639 Neurorehabil. Neural Repair 30, 963-971.

640

641 Maidan, I., Shustak, S., Sharon, T., Bernard-Elazari, H., Geffen, N., Giladi, N., Hausdorff, J.M., 642 Mirelman, A., 2018. Prefrontal cortex activation during obstacle negotiation: what's the effect 643 size and timing? Brain Cogn. 122, 45-51.

644

645 Meester, D., Al-Yahya, E., Dawes, H., Martin-Fagg, P., Piñon, C., 2014. Associations between 646 prefrontal cortex activation and H-reflex modulation during dual task gait. Front. Hum. Neurosci. $6478,78$.

648

649 Mirelman, A., Maidan, I., Bernad-Elazari, H., Nieuwhof, F., Reelick, M., 2014. Increased frontal 650 brain activation during walking while dual tasking: an fNIRS study in healthy young adults. J.

651 Neuroeng. Rehabil. 11, 85.

652

653

654

655

656

657

658

659

660

661 Montero-Odasso, M., Verghese, J., Beauchet, O., Hausdorff, J.M., 2012. Gait and cognition: a

662 complementary approach to understanding brain function and the risk of falling. J. Am. Geriatr. 663 Soc. 11, 2127-2136.

664

665

666

Mirelman, A., Maidan, I., Bernad-Elazari, H., Shustack, S., Giladi, N., Hausdorff, J.M., 2017. Effects of aging on prefrontal brain activation during challenging walking conditions. Brain Cogn. 115, 41-46.

Moher, D., Liberati, A., Tetzlaff, J., Altman, D.G., PRISMA Group, 2009. Preferred reporting items for systematic reviews and meta-analyses: The PRISMA statement. PLoS Med. 6, e1000097.

667 
668 Naito, E., Hirose, S., 2014. Efficient foot motor control by Neymar's brain. Front. Hum.

669 Neurosci. 8, article 594.

670

671 Nieuwhof, F., Reelick, M.F., Maidan, I., Mirelman, A., Hausdorff, J.M., Rikkert, M.G.M.O.,

672 Bloem, B.R., Muthalib, M., Claassen, J.A.H.R., 2016. Measuring prefrontal cortical activity

673 during dual task walking in patients with Parkinson's disease: feasibility of using a new portable

674 fNIRS device. Pilot Feasibility Stud. 2, 59.

675

676 Obrig, H., Villringer, A., 2003. Beyond the visible - imaging the human brain with light. J.

677 Cereb. Blood Flow Metab. 23, 1-18.

678

679 Osofundiya, O., Benden, M.E., Dowdy, D., Mehta, R.K., 2016. Obesity-specific neural cost of 680 maintaining gait performance under complex conditions in community-dwelling older adults.

681 Clin. Biomech. 35, 42-48.

682

683 Raz, N., Kennedy, K.M., 2009. A systems approach to the aging brain: Neuroanatomic hanges, 684 their modifiers, and cognitive correlates. Imaging the aging brain. Jagust, W., D’Esposito, M., 685 New York, NY, Oxford University Press: 43-70.

686

687 Reuter-Lorenz, P.A., Cappell, K.A., 2008. Neurocognitive aging and the compensation 688 hypothesis. Curr. Dir. Psychol. Sci. 17, 177-182.

689

690

691

692 Stuart, S., Vitorio, R., Morris, R., Martini, D.N., Mancini, M., 2018. Cortical activity during 693 walking and balance tasks in older adults and in people with Parkinson's disease: a structured 694 review. Maturitas 113, 53-72.

695

696 Suzuki, M., Miyai, I., Ono, T., Oda, I., Konishi, I., Kochiyama, T., Kubota, K., 2004. Prefrontal 697 and premotor cortices are involved in adapting walking and running speed on the treadmill: an 698 optical imaging study. Neuroimage 23, 1020-1026.

699

700 Suzuki, M., Miyai, I., Ono, T., Kubota, K., 2008. Activities in the frontal cortex and gait

701 performance are modulated by preparation. An fNIRS study. Neuroimage 39, 600-607.

702

703 Takakusaki, K., Tomita, N., Yano, M., 2008. Substrates for normal gait and pathophysiology of 704 gait disturbances with respect to the basal ganglia dysfunction. J. Neurol. 255, 19-29.

705 
706 Takeuchi, N., Mori, T., Suzukamo, Y., Tanaka, N., Izumi, S., 2016. Parallel processing of

707 cognitive and physical demands in left and right prefrontal cortices during smartphone use while 708 walking. BMC Neurosci. 17, 9.

709

710 Tamnes, C.K., Walhivd, K.B., Dale, A.M., Ostby, Y., Grydeland, H., Richardson, G., Westlye, 711 L.T., Roddey, J.C., Hagler, D.J.Jr, Due-Tonnessen, P., Holland, D., Fjell, A.M., Alzheimer's

712 Disease Neuroimaging Initiative, 2013. Brain development and aging: overlapping and unique

713 patterns of change. Neuroimage 68, 63-74.

714

715 Thumm, P.C., Maidan, I., Brozgol, M., Shustak, S., Gazit, E., Shiratzki, S.S., Bernard-Elazari, 716 H., Beck, Y., Giladi, N., Hausdorff, J.M., Mirelman, A., 2018. Treadmill walking reduces 717 prefrontal activation in patients with Parkinson's disease. Gait Posture 62, 384-387.

718

719 Verghese, J., Wang, C., Ayers, E., Izzetoglu, M., Holtzer, R., 2017. Brain activation in high720 functioning older adults and falls: prospective cohort study. Neurology 88, 191-197.

721

722

723

Vitorio, R., Stuart, S., Rochester, L., Alcock, L., Pantall, A., 2017. fNIRS response during

724 walking - artefact or cortical activity? A systematic review. Neurosci. Biobehav. Rev. 83, 160-

725

726

727 172 .

728

729

West, R.L., 1996. An application of prefrontal cortex function theory to cognitive aging.

Psychol. Bull. 120, 272-292.

730

Wu, Z., Mazzola, C.A., Catania, L., Owoeye, O., Yaramothu, C., Alvarez, T., Gao, Y., Li, X.,

731

732 2018. Altered cortical activation and connectivity patterns for visual attention processing in young adults post-traumatic brain injury: a functional near infrared spectroscopy study. CNS

733

734 Zimeo-Morais, G.A., Scholkmann, F., Balardin, J.B., Furucho, R.A., De Paula, R.C.V., Biazoli735 Jr, C.E., Sato, J.R., 2018. Non-neuronal evoked and spontaneous hemodynamic changes in the 736 anterior temporal region of the human head may lead to misinterpretations of the functional near737 infrared spectroscopy signals. Neurophotonics 5, article 011002. 
Figure 1

Figure 1: Flowchart of the articles selection process.

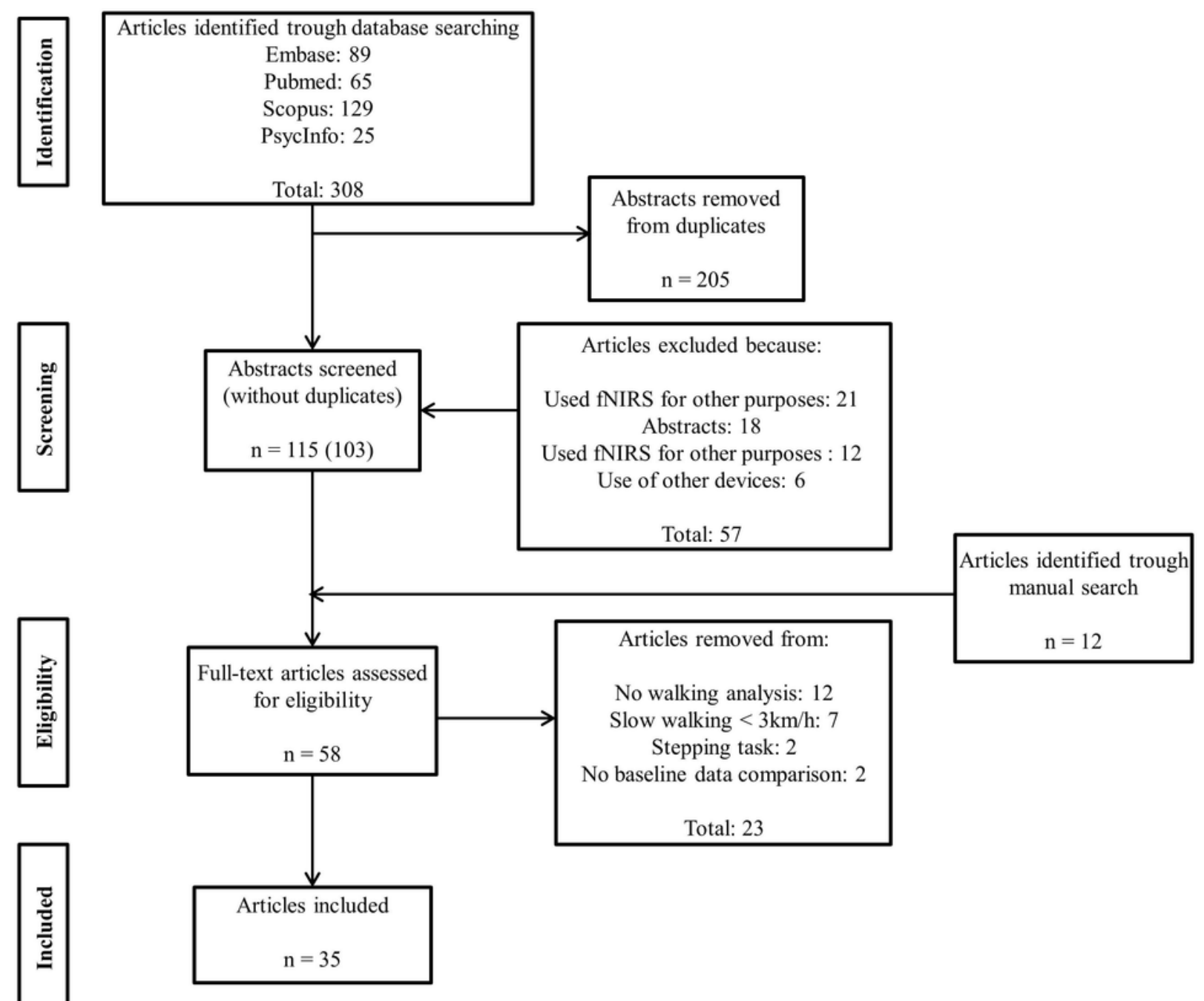




\section{Figure 2}

Figure 2: Proportion of comparisons within each group showing increase (up arrows), decrease (down arrows) or no change (circle) in Prefrontal Cortex (PFC) activation when comparing walking with secondary task versus baseline.

$$
\begin{gathered}
\text { Healthy } \\
\text { young people, } n=20
\end{gathered}
$$

כִ

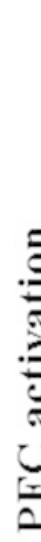

$$
\text { 西 }
$$

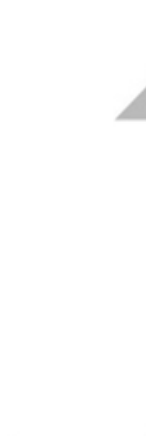

$55 \%$
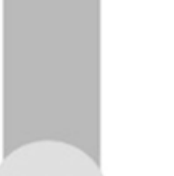

\section{Healthy \\ older people, $n=37$}

$20 \%$

$33 \%$

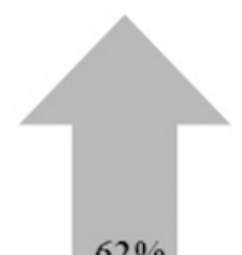

$25 \%$
Clinical

Groups, $\mathrm{n}=18$

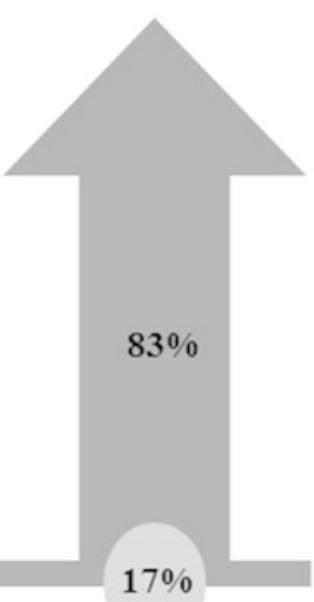




\section{Table $\mathbf{1}$ (on next page)}

Table 1: Summary of fNIRS studies.

PFC: Prefrontal cortex; DT: dual-task; OxyHb: oxygenated hemoglobin; DeoxyHb: deoxygenated hemoglobin; fNIRS: functional near-infrared spectroscopy; TOI: tissue oxygenated index; MS: Multiple Sclerosis; HOA: healthy older adults; MCl: mild cognitive impairment; PMC: premotor cortex; SMA: supplementary motor area; SMC: sensorimotor cortex; S1: primary sensorimotor cortex; M1: primary motor cortex; Hb: hemoglobin; BA: Brodmann area; PD: Parkinson's disease. 


\begin{tabular}{|c|c|c|c|c|c|c|c|c|c|c|c|}
\hline Author, year & Sample & Aims & Gait assessment & Secondary task & Equipment details & $\begin{array}{l}\text { Measurable } \\
\text { parameters }\end{array}$ & $\begin{array}{l}\text { Brain } \\
\text { areas }\end{array}$ & $\begin{array}{l}\text { Controlling, artefacts } \\
\text { and filtering }\end{array}$ & Results & Limitations & Conclusion \\
\hline Al-Yahya et al., 2016 & $\begin{array}{l}19 \text { individuals with } \\
\text { chronic stroke, } 00 \pm \\
15 \text { years old, } 2 \pm \\
\text { women, } 20 \text { healthy } \\
\text { controls, } 54 \pm 9 \\
\text { years old, } 8 \text { women. }\end{array}$ & $\begin{array}{l}\text { To investigate PFC } \\
\text { activation and } \\
\text { relationshipsp between } \\
\text { PFC activation and gait } \\
\text { measures while walking } \\
\text { under single-task and DT } \\
\text { condition in individuals } \\
\text { with stroke and healthy } \\
\text { controls. }\end{array}$ & $\begin{array}{l}\text { Walking on a treadmill } \\
\text { at self-selected speed (5 } \\
\text { 30s trials). Gait } \\
\text { spatiotemporal } \\
\text { parameters estimated } \\
\text { with inverted pendulum } \\
\text { t model using kinematic } \\
\mathrm{g} \text { data from inertial sensor } \\
\mathrm{T} \text { attached at the level of } \\
\text { the fourth lumbar } \\
\text { vertebra (close to the } \\
\text { centre of masss. } \\
\text { Additional single task: } \\
\text { counting while standing } \\
\text { Baseline: unclear. }\end{array}$ & $\begin{array}{l}\text { Counting backward in } 7 \\
\text { from a random number } \\
\text { between } 291 \text { and } 299 \\
\text { while walking }(5 \text { trials) } \\
\text { for 30s. The outcomes } \\
\text { were: rate and accuracy } \\
\text { of correct answers. . . } \\
\text { advice given as to which } \\
\text { task to prioritize during } \\
\text { DT-walking. }\end{array}$ & $\begin{array}{l}\text { Oxymon Mk III system, } \\
\text { (Artinis Medical } \\
\text { Systems). Wavelengths: } \\
782 \text { and } 859 \mathrm{~nm} .8 \\
\text { channels. Interoptode } \\
\text { position } 3.0 \mathrm{~cm} \text {. } \\
\text { Sampling rate: } 10 \mathrm{~Hz} \text {. }\end{array}$ & $\begin{array}{l}\text { OxyHb and } \\
\text { deoxyHb analyed } \\
\text { during } 10 \text { window } \\
\text { between } 6 \text { and } 165 \\
\text { post stimulus onset. }\end{array}$ & PFC. & $\begin{array}{l}\text { Blood pressure was } \\
\text { measured at the beginning } \\
\text { and after the end of each } \\
\text { trial. To remove high- } \\
\text { frequency noise (cardiac } \\
\text { pulsation) fNIRS signals } \\
\text { were then low-pass filtered } \\
\text { at } 0.67 \mathrm{~Hz} \text { cut-off } \\
\text { frequency. }\end{array}$ & 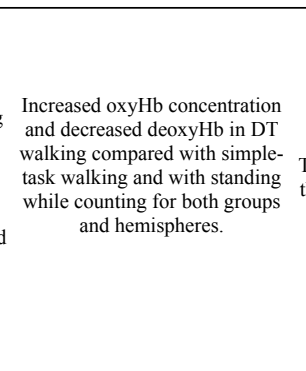 & $\begin{array}{l}\text { The authors pointed } \\
\text { the sample size as a } \\
\text { limitation. }\end{array}$ & $\begin{array}{c}\text { Increased PFC activation } \\
\text { in DT walking versus } \\
\text { single task, among stroke } \\
\text { patients. } \\
\text { No between-group } \\
\text { differences in PFC } \\
\text { activation during walking. }\end{array}$ \\
\hline Beurskens et al., 2014 & $\begin{array}{l}15 \text { young adults, } 25 \pm \\
3 \text { years, } 10 \text { HOA }, 71 \\
\pm 4 \text { years old. }\end{array}$ & $\begin{array}{l}\text { To compare the effects } \\
\text { of completitig a } \\
\text { secondary visual } \\
\text { checking task versus a } \\
\text { verbal memory task } \\
\text { during walking on PFC } \\
\text { activation in young and } \\
\text { HOA. }\end{array}$ & $\begin{array}{c}\text { Walking on a treadmill } \\
\text { at self-selected speed (2 } \\
\text { 30s trials per condition). } \\
\text { Baseline condition: rest } \\
\text { period seating on a } \\
\text { chair. Gait outcomes: } \\
\text { step duration, step } \\
\text { length and number of } \\
\text { steps. }\end{array}$ & 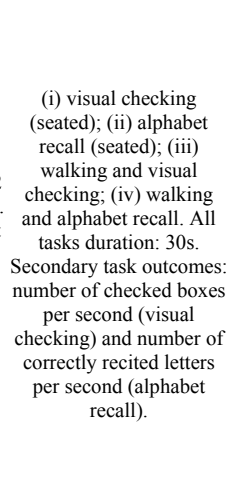 & $\begin{array}{l}\text { DYNOT Imaging } \\
\text { System, NIRx Medical } \\
\text { Technologies, LLC. } \\
\text { Wavelengths: } 760 \text { nm } \\
\text { and } 830 \text { n.m. } 14 \\
\vdots \text { channels. Interoptede } \\
\text { positition: } 2.2 \text { and } 2.5 \\
\text { cm. Sampling rate: } \\
1.81 \mathrm{~Hz} \text {. } \\
\\
\end{array}$ & $\begin{array}{l}\text { OxyHb and } \\
\text { deoxyHb. }\end{array}$ & PFC. & $\begin{array}{l}\text { Tasks were conducted in a } \\
\text { dimly illuminated room. } \\
\text { Each channel was visually } \\
\text { inspected and movement } \\
\text { artefacts were corrected } \\
\text { and data were } \\
\text { reconstructed. } \\
\text { Hemodynamic response } \\
\text { function low pass filter } \\
\text { and wavelet-minimum } \\
\text { description length de- } \\
\text { trending algorithm to } \\
\text { remove possible global } \\
\text { trends due to breathing, } \\
\text { heartbeat vasoconstriction } \\
\text { or experimental } \\
\text { influences. }\end{array}$ & 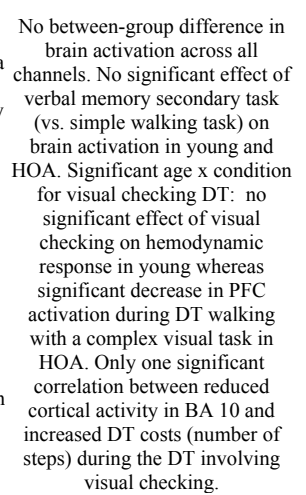 & & $\begin{array}{l}\text { Likely shiff of processing } \\
\text { resources from the PिC to to } \\
\text { other brain regions (not } \\
\text { analysed in this study) } \\
\text { when HOA Aaced he } \\
\text { challenge of walking and } \\
\text { concurrently executing a } \\
\text { visually demanding task. }\end{array}$ \\
\hline Caliandro et al., 2015 & $\begin{array}{l}19 \text { individuals with } \\
\text { chronic gait ataxia, } \\
31-170 \text { years old, , } 10 \\
\text { women, } 15 \text { health } \\
\text { controls, 36-76 yeyars } \\
\text { old, } 8 \text { women. }\end{array}$ & 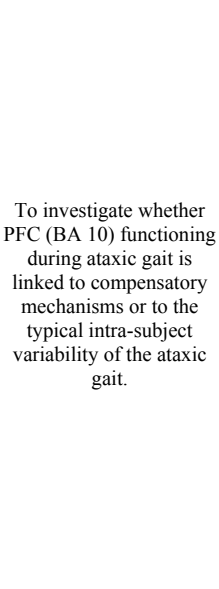 & 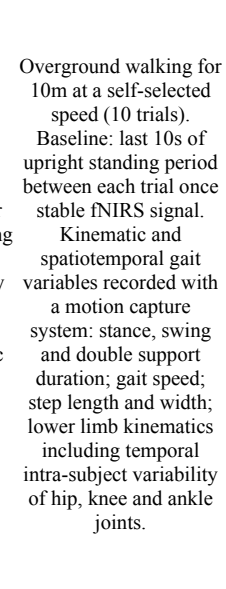 & 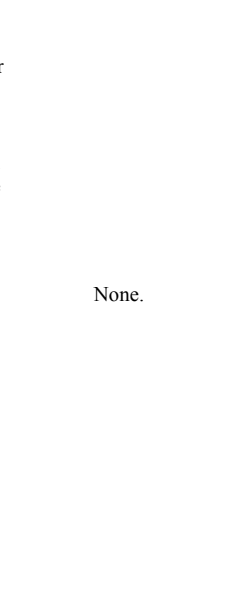 & $\begin{array}{l}\text { NIRO-200, Hamatsu } \\
\text { Phtonics KK. } \\
\text { Wavelengths: } 775 \mathrm{nnm}, \\
\text { 810nmand } 850 \mathrm{~nm} .2 \\
\text { channels. Interoptode } \\
\text { position: 4cm. } \\
\text { Sampling rate: } 2 \mathrm{~Hz} .\end{array}$ & $\begin{array}{c}\text { OxyHb, deoxyHb } \\
\text { and TOI. }\end{array}$ & PFC. & 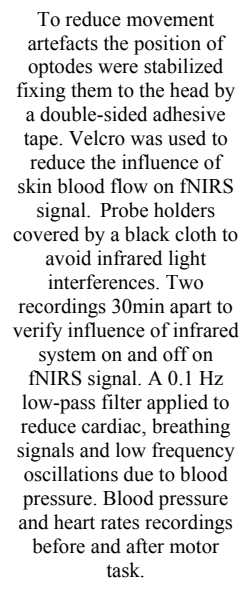 & 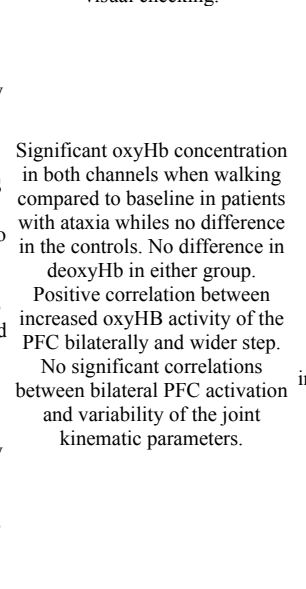 & $\begin{array}{l}\text { Small sample size } \\
\text { which is } \\
\text { acknowledged by } \\
\text { the author. } \\
\text { Influence of skin } \\
\text { blood flow on } \\
\text { fNIRS signals } \\
\text { especially as } \\
\text { interoptode distance } \\
\text { is high (4cm). }\end{array}$ & $\begin{array}{l}\text { Increased PFC activity } \\
\text { during walking (versus } \\
\text { standing) is associated } \\
\text { with increased step width } \\
\text { and therefore it might be } \\
\text { involved in maintaining } \\
\text { compensatory mechanisms } \\
\text { rather than be correlated } \\
\text { with primary defective } \\
\text { cerebellar control. }\end{array}$ \\
\hline
\end{tabular}


10 indidats wo examine the effects $\begin{gathered}\text { body-weight support } \\ \text { conditions). Self-paced }\end{gathered}$ $\begin{array}{llll}\text { fNIR Imager 1000, } & \\ \text { (fNIR Devices LLC }\end{array}$ Potomac, MD)
. and $12 \mathrm{HOA}$ (mean $63 \pm$ yyears, ctivation while DT in MS. (number of correct Standing still and

time). Interoptode positi Sampling rate: $2 \mathrm{~Hz}$. head, $10 \mathrm{~s}$ before each condition. Gait outcomes: gait speed, tride length, stride time
and stride frequency.
$\mathrm{OxyHb}$

1 trial in each condition (i) simple walking; (ii) (instruction to pay Walk on an electronic
pathway at their selfTo examine the changes selected pace for three $90 \mathrm{HOA}, 78 \pm 5$ during obstacle

Chen et al., 2017 years old, 46 negotiation under sing HOA. tasks; recite alternate (iil) ind counting silently in obstacle negotiation the head at a rate of one elliptical shape); (iv) number per second. Gait obstacle negotiation
outcome: stride velocity, during walking while talk outcome: stride velocity. during walking while talk
(same instructions as for (same instructions as for
walking while talking). fNIR Imager 1000,
(fNIR Devices LLC,
Potomac, MD) 16 channels. Interoptode position: Sampling rate: $2 \mathrm{~Hz}$
MS participants exhibited higher activation patterns in all conditions (i.e., task and body weight supportt) when compared PFC activation in DT compat with single walking task. Task cohort interaction whereby greater PFC activation level in MS in DT compared to control. Cohort x support condition showed greater activation in partial body-weight support compared with MS. Cohort $x$ task Raw data visually inspected for excessive support condition whereby MS patients showed higher PFC activation in non- body-weig
support condition in DT PFC. Low-pass filter with a cut- compared with the controls. move the physiological ffects and any addition nois

between gait parameters and oxyHb levels. Similar levels of activation during the last $10 \mathrm{~s}$ when compared to the first $10 \mathrm{~s}$ walking in partial body-weigh support and in MS during the DT partial body-weight support trials; this indicates maintenance of PFC activation levels across he trial. Significant time $x$ task $x$ interaction provided, HOA increased PFC ctivation across time while $\mathrm{MS}$

Data were visually spected and removed in case of saturation or dark

PFC oxyHb levels significantly task irrespective of obstacle
tol a cut-off frequency at 0.14 condition. Slow gait moderated the increase in PFC activation in
tbstacle conditions: compared FFC. respiration and heart rate nals and unwanted hig participants with normal gait dditional frequency noise
was identified and woved by an expert dat$$
\text { analyst. }
$$
ifficulty increased and partia body-weight support was patterns. PFC activation levels were significantly increased in

MS patients unable to No reporting on activation levels across D light, walking condition, unles tide Authors the use of partial bodynowledge small weight support may cause mple sizes and a therapeutic effect, which allows individuals with limitations in physical PFC activation levels. simple walking.

No reporting of controlling for
external light. The authors also
Individuals with mobility limitations (slow gait)
utilized more cognitive limitation as well resources when navigating s that other brain reas involved with obstacle negotiation
were not analysed. 


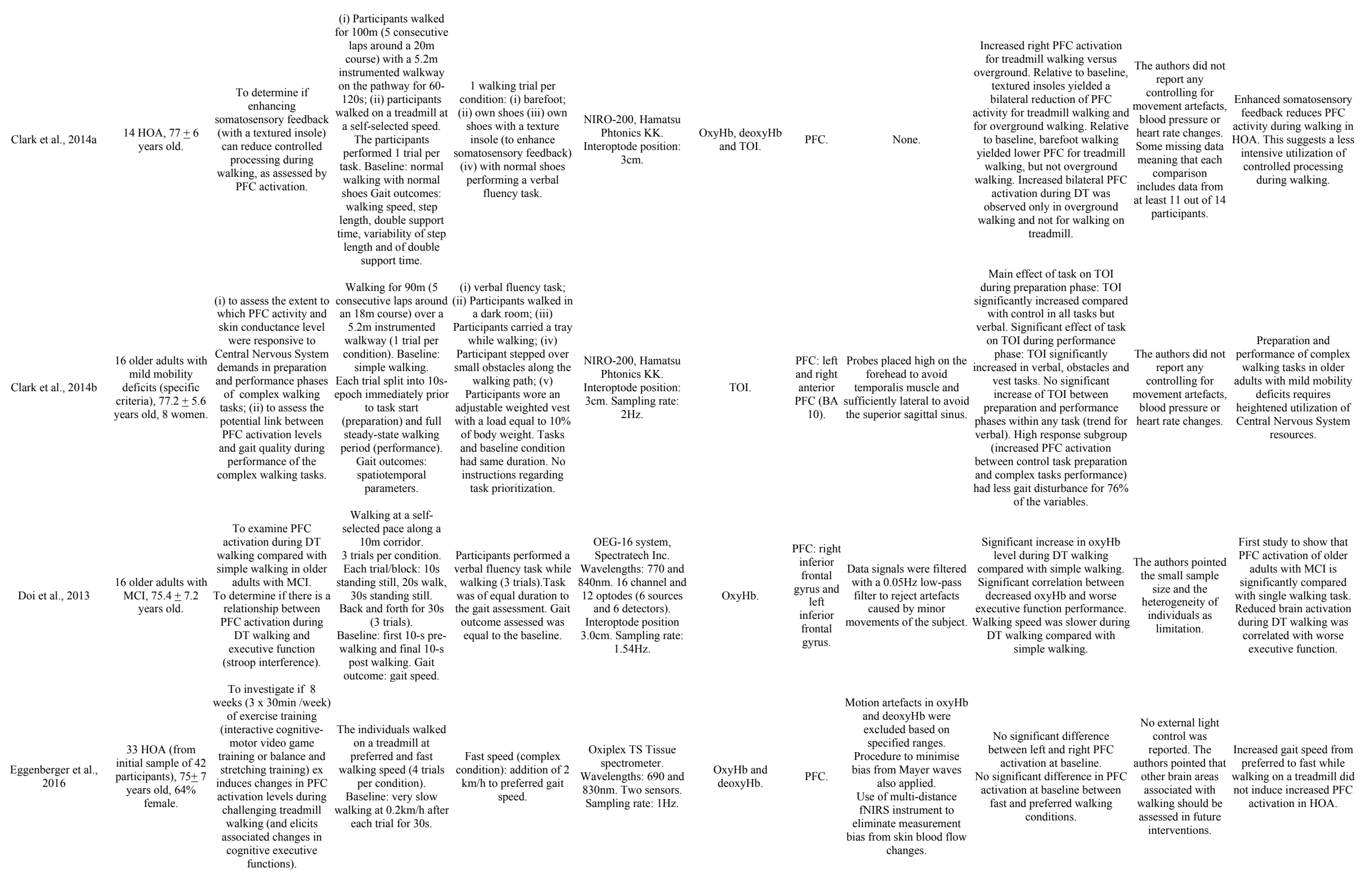




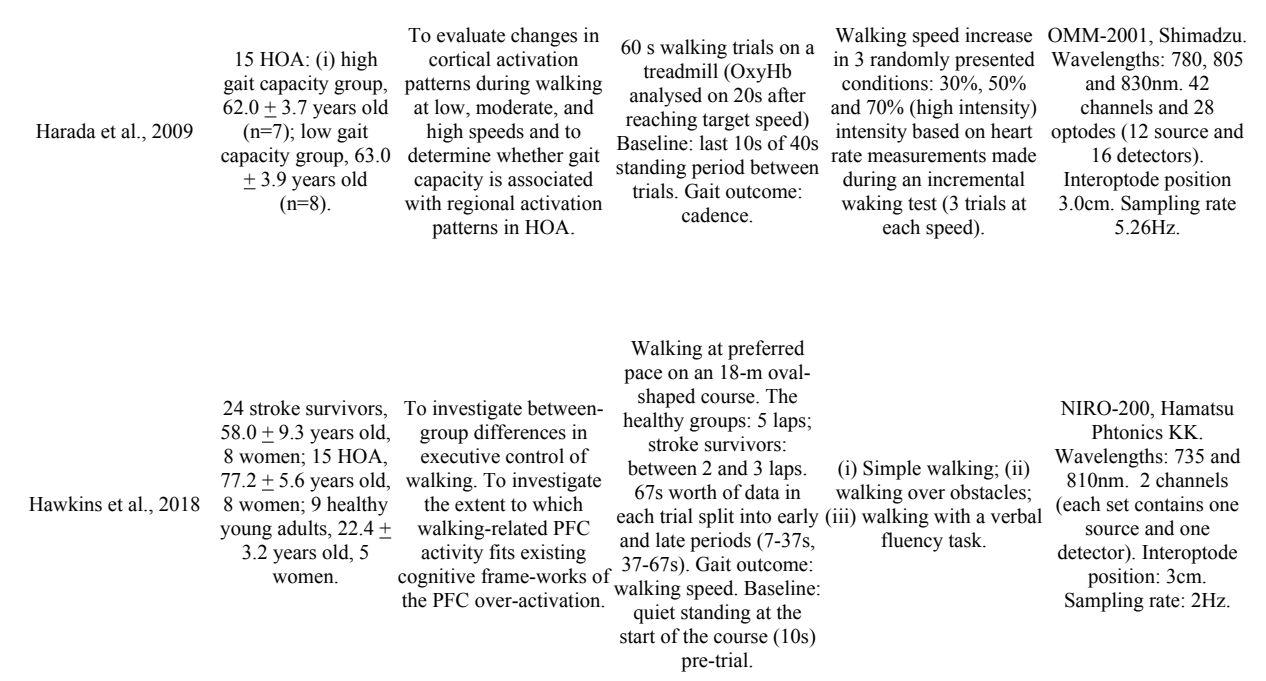

Walking while talking:
Walking at normal pace To investigate the levels $\begin{gathered}\text { along an electronic } \\ \text { pathway at self-selected the alphabet while }\end{gathered}$ 8 individuals with of PFC activation during $\begin{gathered}\text { pathway at self-selected } \\ \text { speed for } 45 \text { metres in alking. Instruction to } \\ \text { pay equal attention to }\end{gathered}$ Devices LLC. $\begin{array}{ccc}\text { MS, } 57 \pm 5 \text { years } & \text { gait under single and DT } \\ \text { conditions in } & \begin{array}{c}\text { speed for } 45 \text { metres in a } \\ \text { continuous looping }(1\end{array} \\ \text { trial }) \text {. }\end{array}$ Hernandez et al,, 2016 old, 6 women and 8 4 years old 6
women. individuals with and without MS usin

Baseline: 10 s standing trial with participants
counting silently in the head. Gait outco
speed.

Tasks and baseline Tasks and baseline assessment. The outcomes were: correct
letter rate and errors per minute.

\section{Half of the participants} cousy) and the other by 1

To determine whether

Hill et al., 2013 $\begin{array}{cc}12 \text { healthy young } & \begin{array}{c}\text { incremental cognitive } \\ \text { adults aged } 18-22\end{array} \\ \text { workload could be }\end{array}$ years old.
Wavelength: 730,805 and 16 optodes (4 etectors). Interoptode position: $2.5 \mathrm{~cm}$. Sampling rate: $2 \mathrm{~Hz}$. optodes. detected both
behaviourally and $\mathrm{v}$ fNIRS.

\section{of participants counted} Walking for 7.6m (15 $\begin{gathered}\text { of participants counted } \\ \text { backwards by } 7 \text { (difficult) Imager 1000, fNIR }\end{gathered}$ trials). Baseline: $10 \mathrm{~s}$
standing still. Gait standing still. Gai
outcome: gait speec while walking. Tasks an baseline condition of equal duration to the gail
assessment. The outcome was: counting
performance.
7 regions of the left PFC and the SMA during left and Participants' heart rate was $50 \%$ or $30 \%$. Increased activation right PFC, measured during walking in the medial SMC and SMA was left and and their blood pressure correlated with increased gait
right

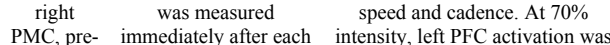
PMC, pre- immediately after each intensity, left PFC activation was SMA and walking task. $\begin{gathered}\text { greater in low gait capacity grou } \\ \text { than in high gait capacity group }\end{gathered}$ medial
SMC. (gait speed $>6 \mathrm{~km} / \mathrm{h}$ at $70 \%$

Significant effect of group for PFC activity during simple an obstacle walking tasks, with All data were inspected for healthy young group exhibiting
signal artefact:
the lowest level of PFC activity signal artefact: the lowest level of PFC activity,
automatically identified as followed by the HOA group and axyHb signal exceeding $1 \mathrm{uM}$ within a 2-second groups, significantly greater

The lighting in he room was controlled for:

approximatively 150 lx or

$1 / 3$ of typical office Individuals with MS had greater lighting To eliminate elevations in PFC oxyHb level tion, heart in walking while talking task

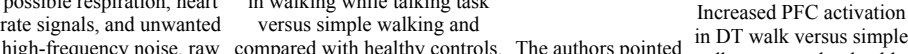

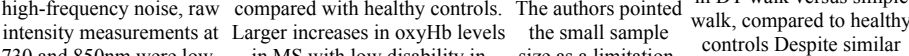
730 and $850 \mathrm{~nm}$ were low- in MS with low disability in size as a limitation. controls Despite similar pass filtered with a finite comparison to high disability gait speed and cognitive whil a cut-of frequency of group walking slower yet not $0.14 \mathrm{~Hz}$. Motion artefacts significantly.

remaining were eliminated

PFC by visual inspection of an

(BA 9 and A low-pass filter was Significantly greater oxyHb level 46) applied to eliminate in PFC in the difficult versus Higher level of PFC anterior rate, respirtion acter collapsed across optodes Movement artefacts activity during a high load PFC (BA unwanted noise signals. locations. Cortical activation were not controlled condition was observed, 10), and Motion artefact rejection levels in the left-hemisphere were in this study, only which was more part of routine applied to significantly higher in the removed by pronounced in the lef inferior eliminate uninterpretable difficult versus easy condition;
Left PFC is involved in the e-related decline in gait

Young adults have more emaining PFC resources walking conditions and/or secondary cognitive tasks
during walking. There is a executive control resources in HOA and stroke survivors during size of one group walking. The level of PFC $\begin{array}{ll}\text { was }<10 & \text { particularly during }\end{array}$ walking speeds are complex walking tasks
different. walking impairments. of available resources for
individuals who have 


\begin{tabular}{|c|c|c|c|c|c|}
\hline Holtzer et al,, 2016 & $\begin{array}{c}\text { (i) } 167 \text { HOA with no } \\
\text { gait impairments, } \\
74.4 \pm 6.0 \text { years old, } \\
85 \text { women; (ii) } 40 \\
\text { older adults with } \\
\text { peripheral } \\
\text { neurological gait } \\
\text { abnormalities, } 77.0 \\
\pm 6.3 \text { years old, } 17 \\
\text { women; (iii) } 29 \\
\text { older adults with } \\
\text { central neurological } \\
\text { gait abnormalities, } \\
79.6 \pm 7.4 \text { years old, } \\
20 \text { women. }\end{array}$ & $\begin{array}{l}\text { To determine the effect } \\
\text { of neurological gait } \\
\text { abnormalities on the } \\
\text { functional neural } \\
\text { correlates of locomotion } \\
\text { in older adults with } \\
\text { regards to the posture } \\
\text { first hypothesis. }\end{array}$ & $\begin{array}{l}3 \text { continuous loops of } \\
\text { walking on an electronic } \\
\text { pathway }(6 \text { straight line } \\
\text { trials of } 4.5 \mathrm{~m}-\text {-long }) \text { at } \\
\text { self-selected speed. } \\
\text { Baseline: } 10 \mathrm{~s} \text { standing } \\
\text { trial pre-walk, counting } \\
\text { silently in the head at } \\
\text { rate of one number per } \\
\text { second. Gait outcome: } \\
\text { stride velocity. }\end{array}$ & $\begin{array}{l}\text { Reciting alternate letters } \\
\text { of the alphabet while } \\
\text { walking } 3 \text { continuous } \\
\text { loops as in the single } \\
\text { walking task }(6 \text { straight } \\
\text { line trials of } 4.5 \mathrm{~m} \text {-long). } \\
\text { Instruction to pay equal } \\
\text { attention to their walking } \\
\text { and talking. Outcomes: } \\
\text { letter rate and errors per } \\
\text { minute. }\end{array}$ & $\begin{array}{l}\text { Imager } 1000 \text { (fNIRS } \\
\text { Devices LLC, Potomac } \\
\text { MD). Wavelength: } 730 \text {, } \\
\text { (805 not used) and } \\
850 \mathrm{~nm} \text {. } 16 \text { channels. } \\
\text { Interoptode position: } \\
2.5 \mathrm{~cm} \text {. Sampling rate: } \\
2 \mathrm{~Hz}\end{array}$ \\
\hline
\end{tabular}

3 continuous loops of Reciting alternate letter

To determine whether walking on an electronic of the alphabet while Imager 1000 (fNIRS

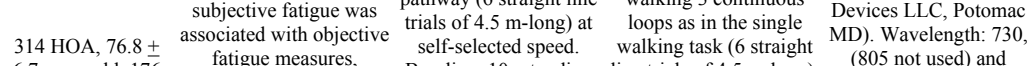

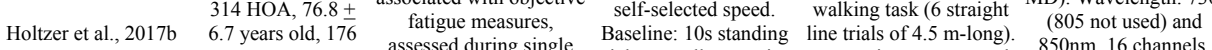

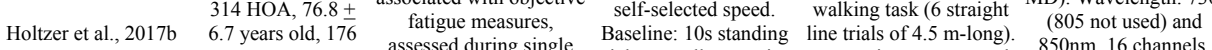
and attention-demanding trial pre-walk, counting Instruction to pay equal $\begin{gathered}\text { Interoptode position: } \\ \text { silently in the head at }\end{gathered}$ $\begin{array}{cccc}\text { DT walking conditions, } & \begin{array}{c}\text { silently in the head at } \\ \text { rate of one number per }\end{array} \text { and talking. Outcomes: } & 2.5 \mathrm{~cm} \text {. Sampling rate } \\ \text { in HOA. } & \text { r thz }\end{array}$ rate of one number per and talking. Outcomes:
second. Gait outcome: letter rate and errors per stride velocity.

To understand the neural Walking on a treadmill mechanism of precision at $3 \mathrm{~km} / \mathrm{h}$ for $35 \mathrm{~s}(10$ 11 healthy young adults, $23 \pm 4$ years $\begin{array}{ll}\text { with normal gait. Also, } 35 \mathrm{~s} \text { quiet standing prio } & \end{array}$ to evaluate the role of to each walking trial. the neural control of gait Gait outcome: step tin
Oxymon (Artinis Medical System,
Zetten the Netherlan the Netherlands) Precision stepping Walking on a treadmil
$3 \mathrm{~km} / \mathrm{h}(10$ trials $)$. nm. 6-channel a 3-channel PFC unit. Interoptode position 1.0
and $4.0 \mathrm{~cm}$. Sampling rate: $25 \mathrm{~Hz}$.
OxyHb.

$\mathrm{OxyHb}$ respiration, heart rate
signals, and unwanted e, raw from normal walking to walking 7hosity meastroments at while talking but it did nor pass filtered with a finite velocity. Worse subjective impulse response filter fatigue did not moderate changes oxyHb during the course of the $0.14 \mathrm{~Hz}$. Saturation were excluded.
war dark curnt condition walk but was associated with attenuated oxyHb levels in
the fourth, fifth and sixth straight the fourth, fifth and sixth straight
walks compared to the first during walk-while-talk.

Second order low-pass Task effect: Increased activation Butterworth int in the significant upper $\mathrm{PFC}$
channel (larger deoxyHb cut-off frequency of do decrease) during the early-task reduce high frequency
compared to normal walking. No noise. A second order compared to normal walking. No high-pass Butterworth signicantintence in motor filter with a cut-off frequency of $0.01 \mathrm{~Hz}$ wa
used to reduce low

OxyHb and
deoxyHb. hemispher
e: S1, M1, frequency drift cause by crices activation between the 2 early or late phase. Phase effect in PFC: normal The authors pointed $\begin{array}{cc}\text { SMA, } & \text { correction for superficial } \\ \text { pre-SMA } & \text { interference, a second }\end{array}$ or early and late-task Buterworth filter wint cut-off frequency of $1 \mathrm{H} z$ was conducted. versus late-task in precision Continuous blood pres separation channels we
used to remove cortices activation: in normal walking, larger pre-task oxyHb compared with early and late-
task. The SMA, M1, and S1 revealed no significan differences between normal walking and precision steppin study.

Neural confirmation of the posture first hypothesis emerged among older adults whose postural and compromised. Increased activation in the PFC during locomotion may have a compensatory unction that is designed to adults with peripheral
a neurological gait abnormalities.

Worse subjective fatigue attenuation in both the increase of oxyHb levels nknown if lighting walking while talking and during the course of walking while talking. The trajectory of oxy $\mathrm{Hb}$ during simple walking, however, subjective fatigue.

The lack of M1/S1 activation during gait
suggests that even in the current precision stepping ask the control of ongoing
gait depended mostly on gait depended mostly on
subcortical automatisms, while motor cortices
contributions did not differ between standing and walking. A prolonged
activation of the PFC for precision stepping indicated that more perform precision stepping in comparison to normal walking. 


\begin{tabular}{|c|c|c|c|c|c|c|c|c|c|c|c|}
\hline Lin \& Lin, 2016 & $\begin{array}{l}24 \text { healthy young } \\
\text { adults, } 20-27 \text { years } \\
\text { old, } 12 \text { women. }\end{array}$ & $\begin{array}{l}\text { To investigate the } \\
\text { influence of cogntive } \\
\text { task complexity and } \\
\text { walking road condition } \\
\text { on the nentral correleates } \\
\text { of executive function and } \\
\text { postural control in DT } \\
\text { walking. }\end{array}$ & 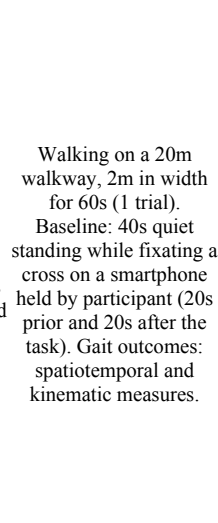 & 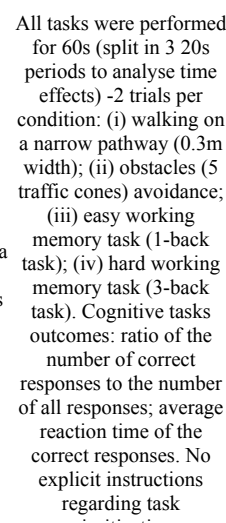 & 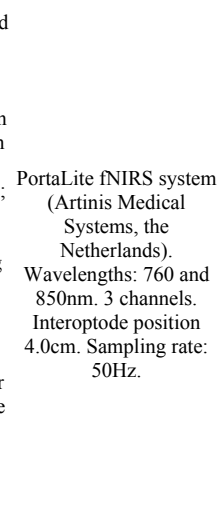 & Охунь. & PFC. & $\begin{array}{l}\text { Data was low-pass filtered } \\
\text { with a finiti impulse } \\
\text { response filter with a cut- } \\
\text { off frequency of } 0.2 \mathrm{~Hz} \text { to } \\
\text { attenuate the noises from } \\
\text { non-evoked neurovascular } \\
\text { coupling. }\end{array}$ & 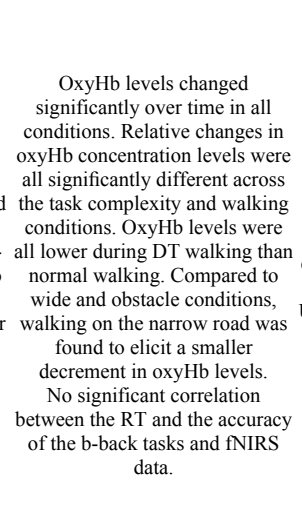 & $\begin{array}{l}\text { No mention of } \\
\text { whether movement } \\
\text { artefects were } \\
\text { controlded for in the } \\
\text { data processing. } \\
\text { Unknown if ilihting } \\
\text { condition were } \\
\text { controlled for. } \\
\text {. }\end{array}$ & $\begin{array}{l}\text { Healthy young adults are } \\
\text { inclined to focus on the } \\
\text { challenging working } \\
\text { memory task and } \\
\text { sacrificed gait } \\
\text { performance to some } \\
\text { extent through } \\
\text { altered neural activations } \\
\text { in the PFC and adapted } \\
\text { coordination of } \\
\text { lower-extremity } \\
\text { kinematics. }\end{array}$ \\
\hline Lu et al., 2015 & $\begin{array}{l}17 \text { heathy young } \\
\text { adults, } 23.1 \pm 1.1 .5 \\
\text { years old, } 8 \text { women. }\end{array}$ & $\begin{array}{l}\text { (i) Te evaluate the } \\
\text { declines in gait } \\
\text { performance caused by } \\
\text { differeing DT } \\
\text { interference; (ii) to } \\
\text { assess the alterations in } \\
\text { cortical activation in the } \\
\text { PFC, PMC and inAA } \\
\text { when walking and } \\
\text { performing a second } \\
\text { cognitive or motor task } \\
\text { compared with walking } \\
\text { at a normal pace; (iii) To } \\
\text { investitiate the } \\
\text { association between } \\
\text { cortical activation and } \\
\text { gait performance during } \\
\text { DT. }\end{array}$ & 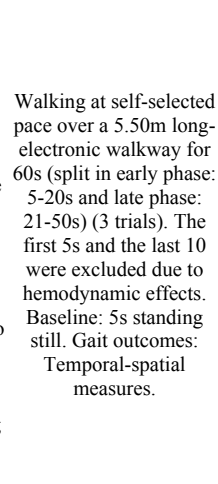 & $\begin{array}{l}\text { 3 trials in each condition, } \\
\text { block-randomised: (i) } \\
\text { walking while performing } \\
\text { a cognitive task } \\
\text { (subtracting } 7 \text { from an } \\
\text { initial } 3 \text {-didigit number); } \\
\text { (ii) walking while } \\
\text { performing a motor task } \\
\text { (carrying a bottle on a } \\
\text { tray). Tasks duration and } \\
\text { baseline condition are } \\
\text { equal the gait assessment. }\end{array}$ & $\begin{array}{l}\text { NIRSport (NIRx } \\
\text { Medical Technologies } \\
\text { LLC, NY, USA). } \\
\text { Wavelengths: } 760 \text { and } \\
\text { 850n. } 14 \text { channels. } \\
\text { Interoptode position: } \\
\text { 3.0cm. Sampling rate: } \\
\text { d } 7.81 \mathrm{~Hz} \text {. } \\
\text { t. }\end{array}$ & $\begin{array}{l}\text { OxyHb, deoxyHb } \\
\text { and index of } \\
\text { haemoglobin } \\
\text { differential oxy } \\
\text { deOxyHb). }\end{array}$ & $\begin{array}{l}\text { PFC, } \\
\text { PMC and } \\
\text { SMA }\end{array}$ & 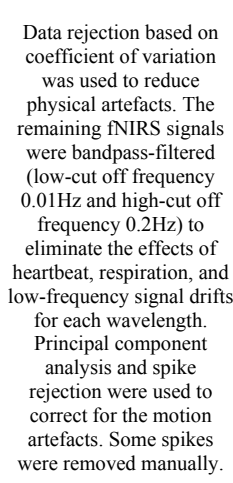 & 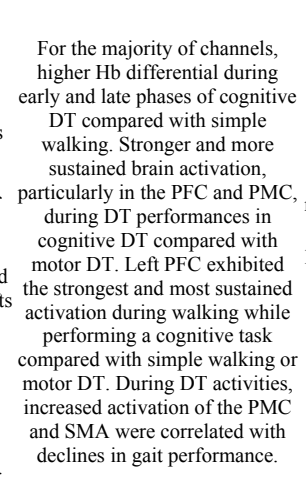 & $\begin{array}{l}\text { The authors did not } \\
\text { record the cognitive } \\
\text { task and motor task } \\
\text { performances in the } \\
\text { DT conditions } \\
\text { compared with the } \\
\text { single tasks. }\end{array}$ & $\begin{array}{l}\text { The negative relationship } \\
\text { between PMC and SMA } \\
\text { activation and gait } \\
\text { variables suggests a } \\
\text { control mechanism for } \\
\text { maintaining gait } \\
\text { performance during DT. }\end{array}$ \\
\hline Lucas et al., 2018 & 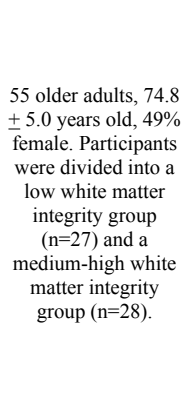 & $\begin{array}{l}\text { To examine the } \\
\text { relationship betwween } \\
\text { white matter } \\
\text { microstructural integrity } \\
\text { and changes in PFC } \\
\text { oxylH during active } \\
\text { walking in older adults. }\end{array}$ & 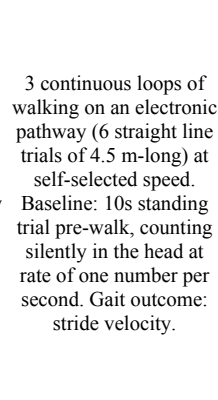 & 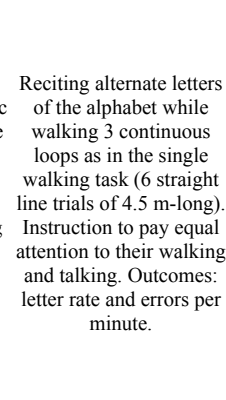 & $\begin{array}{l}\text { Imager } 1000 \text { (fNIIRS } \\
\text { Devices LLC, Potomac } \\
\text { MD). Wavelength: 730, } \\
\text { (805 not usedd and } \\
850 \mathrm{~nm} .16 \text { channels. } \\
\text { Interoptode position: } \\
\text { 2.5cm. Sampling rate: } \\
2 \mathrm{~Hz}\end{array}$ & ОхуНь. & PFC. & 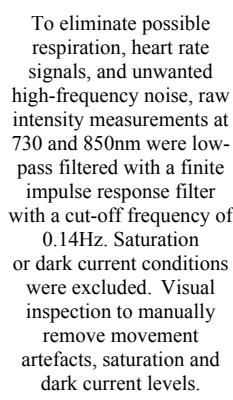 & 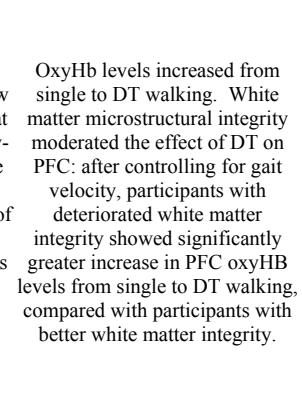 & $\begin{array}{l}\text { No mention of } \\
\text { controlling for } \\
\text { external light. The } \\
\text { authors pointed that } \\
\text { the use of short } \\
\text { source-detector } \\
\text { channels and use of } \\
\text { advanced filtering } \\
\text { s, should be used in } \\
\text { future studies. }\end{array}$ & $\begin{array}{l}\text { Compromised white } \\
\text { matter microstructural } \\
\text { integrity may be a } \\
\text { mechanimu underlying } \\
\text { ineffficient brain response } \\
\text { to increased cognitive } \\
\text { demands of locomotion. }\end{array}$ \\
\hline
\end{tabular}


Meester et al., $2014 \quad$ adults, $27.8 \pm 6.3$

Mirelman et al., 2014

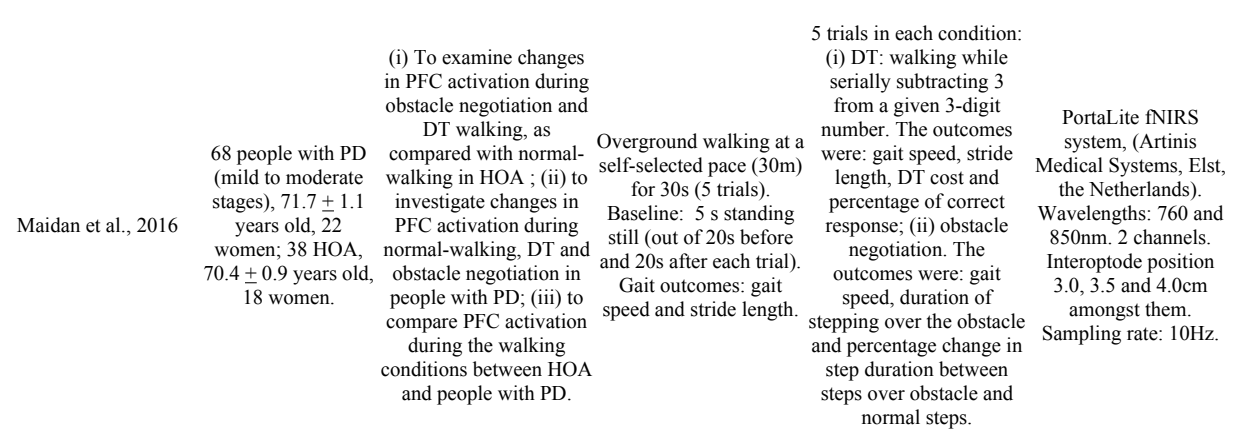

3 trials in each of 4 conditions: anticipated

One trial of overground 20 healthy young To explore the effects of walking on an elliptical years old, 10 obstacle height and available response tim each obstacle. Gait outcomes: gait speed

Maidan et al., 2018 years, ights $(50$ and $100 \mathrm{~mm})$. system, Artinis Medical dition Systems. Wavelength 760 and $850 \mathrm{~nm}$. were the same as the
mple walking. 3 phases of $3 \mathrm{~s}$ analysed: before, over and after obstacles.

Walking on a treadmill at a self-selected pace and $20 \%$ faster for 30 ( processing (PFC activity, without secondary task). 10 s in the middle of the spinal cord activity and Baseline: 10s standing trials used for statistical gait) during walking in still in the middle of resting time between
trials. Gait outcome: analyses. trials. Gait outcom
step time.

(i) walking while 5 trials of walking on a a $\begin{gathered}\text { counting forward; (ii) } \\ \text { walking while serially } \\ \text { subtracting } 7 \text { from a pre- }\end{gathered}$ $30 \mathrm{~m}$ walkway at self- $\begin{gathered}\text { subtracting } 7 \text { from a pre- } \\ \text { determined } 3 \text { digit }\end{gathered}$

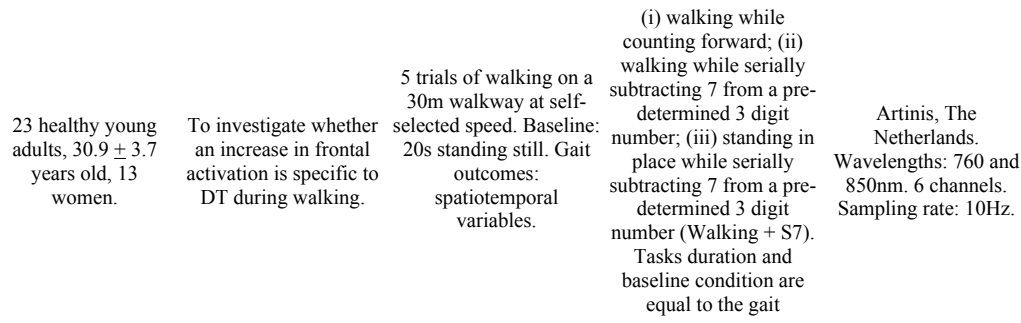

People with PD had significant

higher activation during normal

interaction during DT,

Probes were shielded from increased only in HOA. In ambient light by covering contrast oxyHb increased the forehead with black significantly during obstacle fabric. A bandpass filter negotiation compared with

OxyHb and
deoxyHb. with frequencies of 0.01 physiolos used to reduce physiological noise (drift
of the signal and heart beat). To remove motion . Significantly greater relative
increase in oxyHb during DT artefacts, a wavelet filter was used. compared with usual walking, for was used for that
the HOA compared with the PD proposal. The order participants. In PD, higher PFC of the trials was not activation associated with faster
gait speed. More clinical ymptoms in PD were associated with lower PFC activation in mple walking and obstacle

$$
\begin{aligned}
& \text { conditions. } \\
& \text { cons }
\end{aligned}
$$

PFC activation significantly greater when obstacles compared before and

Probes were shielded from after obstacle crossing. PFC ambient light by covering the forehead with black
fabric. A bandpass filter activation during obstacle
negotiation is not affected by
obstacle height Significant effec with frequencies of 0.01 to ОхуHb.

$0.14 \mathrm{~Hz}$ was ur physiological noise (drift
of the signal and heart beat). To remove motion artefacts, a wavelet
was used.

during unanticipated obstacles, the slope of the oxyHb response was steeper, as compared to anticipated obstacles. These
hanges in PFC activation during changes in PFC activation durin
negotiation of unanticipated
obstacles were correlated with greater distance of the leading foot after the obstacles.

Oxymon (Artinis Netherlands). Netherlands).
Wavelengths: 782 and $859 \mathrm{~nm} .4$ channels. $3.0 \mathrm{~cm}$. Sampling rate: S. years old, cqual to the gait
$\mathrm{OxyHb}$ PFC.
Blood pressure and heart rate were measured at the start and end of testing deoxyHb were calculated and filtered with a low motion artefacts, missing signals and noisy signals. Blocks with missing
signals or artefacts were excluded.

To eliminate hysiologically irrelevant was applied with a finite impulse response filter, with a cut-off frequency at
$0.14 \mathrm{~Hz}$ before processing the signals. and visually inspected for
OxyHb concentrations significantly increased in the a single task walking. PFC activity was unaffected by increases of walking speed. No
significant correlation between ignificant correlation betwee
PFC activation H-reflex variability and step time variability.

Walking alone demonstrated the west levels of oxyHb followed walking + counting condition,
followed by Walking + S7 gnificantly different compar to the two other walking differences in oxyHb levels wer observed between usual walkin and the standing condition or etween standing with or with condition, which was
A different pattern of PFC activation during walking was observed between higher activation channels (2) used in during normal walking in Also, the authors people with PD suggests Ad not control the important role already important role already However, higher activation relative to baseline during obstacle DT in people with PD hows that PFC activation the task.

The authors did not report on channel
numbers and interoptodes thors pointed as a The pattern of PFC use of harness nature of obstacle. During ight have affected unanticipated obstacles the aparis we obstacle the during negotiation of estimate the (action of the same trials.

Healthy young adults The authors pointed increased PFC activity in the use of treadmill response to increasing as a limitation (no ecological
validity) mention of controlling for authors point the high variability of the fNIRS responses betwe erformance and reflex PFC activity allowed
Citity. The incease in individuals to perform additional tasks simultaneously without affecting cortical output
onto the measured peripheral reflexes and thus gait control. The order of the brain activation in healthy young adults. The mention of observed changes are controlling for apparently not a response ambient light artefacts. the cognitive load during 

5 trials of walking on a (i) walking while serially

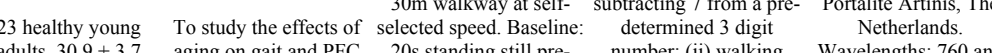
num female; $20 \mathrm{HOA}$, walking task with spatiotemporal variables physical obstacles. Tasks Interoptode distances $\begin{gathered}69.7 \pm 5.8 \text { years old, internal and external task from an electronic mat duration and baseline } \\ 50 \% \text { female. } \\ \text { demands. }\end{gathered}$ placed in the middle of condition are equal to the 35 and 40
$\mathrm{~mm}$. Sampling rate $\begin{array}{ccc}\text { placed in the middle of } & \text { condition are equal to the } & \mathrm{mm} \text {. Sampling rot } \\ \text { the walkway. } & \begin{array}{c}\text { gait assessment. The DT } \\ \text { score was also calculated. }\end{array} & 10 \mathrm{~Hz} \text {. }\end{array}$

Mirelman et al., 20 period. the participants the number 1 -10 in

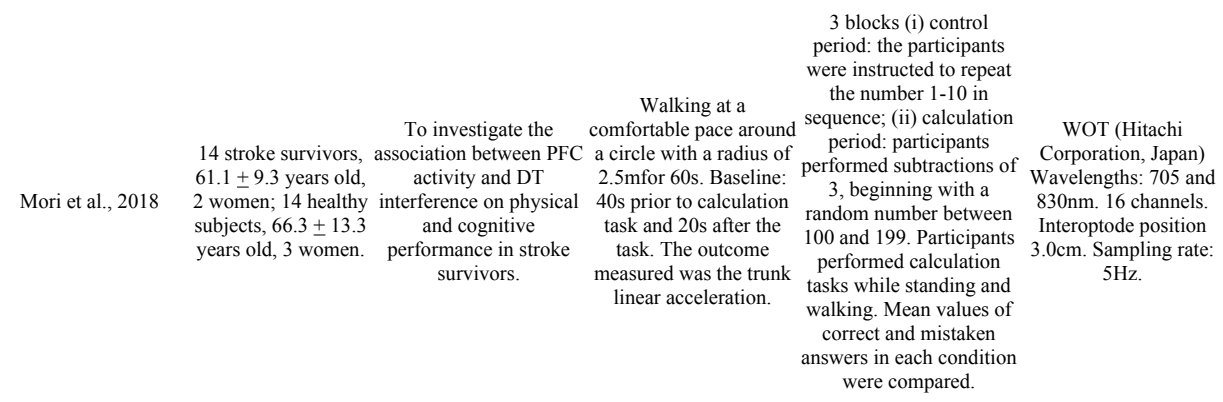

$\mathrm{OxyHb}$

HOA had significant increases in oxyHb levels during simple walking, relative to standing. increased PFC activation in the DT and in the obstacle

A band-pass filter with fequency of $0.01-0.14 \mathrm{~Hz}$ ent was used to reduce group effect whereby younger small sample size; motion artefacts a wavelet
filter was uned, followed
correlation between age and
by correlation based signal by correlation based signal oxyHb levels and gait variability
improvement. Probes were shielded from ambient Age was a significant evels in the usual walking. Age and gait speed were significant levels in the obstacle condition

PFC activation during DT walking was significatly lower in stroke survivors. Right PFC A band pass filter with a activation was negatively A band pass filter with a
low pass $(0.5 \mathrm{~Hz})$ was
correlated with DT cost on
acceleration magnitude in strol applied to account for the acceleration magetitude
effects of Mayer waves survivors, yet not in healthy

PFC. participants. In healthy
articipants, left PFC activation

was significantly negatively
correlated with correct rate an was used for baseline drif. $c$ mistake rate of subtractions. No
significant correlation between significant correlation between
PFC activation and cognitive levels than HOA, in all

ependent predictor of oxyH but not the DT.

$\begin{array}{cc}\text { few probes to } & \text { PFC activation during } \\ \text { analyse the brain } & \text { walking is dependent on }\end{array}$

ectivation in the we and task

C and also, that, HOA apparently rely more

her brain areas on cognitive resources (notor cortex) even during usual walking. should be fiture sudies. ng usual walking

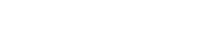

During DT walking, PFC activation might prionitize for light terference. The survivors, but might prioritize cognitive subjects. The results suggest that during DT walking, the stroke $\begin{gathered}\text { responses (i.e. } \\ \text { blood pressure, } \\ \text { heart rate). }\end{gathered}$
motonts demandits while
holth dembents healthy subjects prioritize
the cognitive task. 
(i) To examine the

feasibility of measuring

bilateral PFC activity in

people with PD during
different DT walking

different DT walking

conditions using two 5 trials of walking back
wireless fNIRS devices; and forth over a course

Five blocks each

including each of the
three cognitive task

conditions:(i) walking

作

12 people with $\mathrm{PD}$ a self-selected pace fo walling while serially system, Artini Medic

Nieuwhof et al, 2016 (mild to moderate he after trials (final $5 \mathrm{~s}$ of PFC as consequence of these 20 s periods were rest; (iii) To investigate the sensitivity of the method to detect oxy and deoxyHb

concentrations between
DT walking and simple

walking. Gait outcomes: determined 3 digit git spans. Outcomes: $\quad 3.0,3.5$ and $4.0 \mathrm{~cm}$ the number of amongst them.
ampling rate: $10 \mathrm{~Hz}$ spans completed within

the task and the

percentage of correct
nswers on both task

2 blocks of simple and

complex walking trials.

Each block includes $430-$

long trials (i) walking

Walking back and forth

20 community

To determine the obesity-specific at a self-selected pace cognitive DT (recite

Osofundiya et al., 2016 (ii) 10 obese, $80.5 \pm \begin{array}{r}\text { simple and complex } \\ \text { ambulatory tasks in ol }\end{array}$ .8 years old 6 adults. seline: 30 quiet seating.
quiet stan alphabet) (outcome:
percent correct

percent correct
ponses); (ii) precision
NIRO $200 \mathrm{NX}$ 10 q quiet standing in between blocks. Gait outcome: gait speed. face targets) (outcom

Japan). 2 chonnels. $\mathrm{OxyHb}$ and total $\mathrm{Hb}$

duration

and baseline condition
are equal to the gait

assessment Participants

encouraged to perform

their best on the

OxyHb and
deoxyHb.

\section{PFC}

fNIRS signals were also
linearly de-trended per
trial and low-pass filtered

The devices were shielded from ambient light by
covering the whole

covering the whole
forehead with a black

cloth. The movement

algorithm was performe

within each trial. The

at $0.1 \mathrm{~Hz}$ using a

Butterworth filter to
remove heart rate and

other higher frequency

physiological signals.
Before starting any trial,

Before starting any trial,

participants stood for a

blood pressure fluctuation

after standing up.

Heart rate was
continuously monitored
using a heart rate monitor
and was averaged across
each trial. Participants
instructed to avoid any
sudden head movements
during the tasks. Probes
covered with a black
headband th eliminate
external lights.

Significant task * grou

The authors
Significantly on oxyHe levels.
pointed: small

levels in DT and precision sample size might

walking tasks compared to rest limit the extension In order to maintain gait and usual walking, as well as in obese compared with non-obese were not controlled associated with higher individuals. Obesity was (diabetes, higher neural costs, and this was associated with three times blood pressure, $\begin{array}{lll}\text { during the precisi, particularly } & \text { etc.). Order of ambulatory tasks requiring }\end{array}$ despite obese adults simple and DT trials

external lights. demonstrating similar gait speeds and performances on the complex precision wall gait tasks as non-obese ad

always last.

\section{To assess cortical Three locomotor tasks:}

activation patterns walking at $3 \mathrm{~km} / \mathrm{h}, 5$

Suzuki et al., $2004 \quad \begin{aligned} & 9 \text { healthy young } \\ & \text { adults, } 28.1+7.4\end{aligned}$

associated with

assessed by reelative

changes of oxyHb and
deoxyHb levels using

Increase in locomotor
speed. Task data in the
Japan). Wavelengths:
780,805 and $830 \mathrm{~nm}$

$9 \mathrm{~km} / \mathrm{s}$ on a treadmill for

(1 tral). Baseline:

outcome: cadence. $60 \mathrm{~s}$

13-s period just before

seaching each constan

42 channels.
.

OxyHb, deoxyHb before, 30s after).
Interoptode position
3.0cm. Sampling rate:

$5.26 \mathrm{~Hz}$.
PMC,
medial-
SMC and
lateral-

SMC.
(1)
PFC activation was significantly at $9 \mathrm{~km} / \mathrm{h}$ than when they walked nd arterial oxygen sturation were measured after each task tions in the PFC, PMC a greater than that in the lateral SMC
The PFC was significantly periods before reaching a constant speed in the No mention of $\quad 9 \mathrm{~km} / \mathrm{h}$ run compared with filtering or the $5 \mathrm{~km} / \mathrm{h}$ walk and

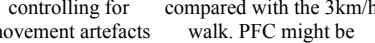
walk. PFC might be
involved together with other structures in controlling locomotion to adapt to the increasing
speed in the acceleration speed in the acceleration
of phase of locomotion. 


\begin{tabular}{|c|c|c|c|c|c|c|c|c|c|c|c|}
\hline Suzuki et al., 2008 & $\begin{array}{c}7 \text { healthy young } \\
\text { adults, 31.3 } \\
\text { years old, } 3 \text { women. }\end{array}$ & $\begin{array}{l}\text { To assess how a verbal } \\
\text { instruction before } \\
\text { walking would affect } \\
\text { corticial activation and } \\
\text { walking performance } \\
\text { using fNIRS. }\end{array}$ & 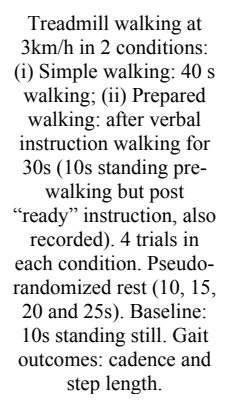 & None. & 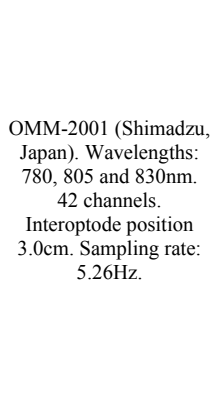 & $\begin{array}{c}\text { OxyHb, deoxyHb } \\
\text { and total Hb levels. }\end{array}$ & $\begin{array}{l}\text { PFC, } \\
\text { SMA, } \\
\text { PMC, } \\
\text { medial } \\
\text { SMC and } \\
\text { lataral } \\
\text { SMC. }\end{array}$ & None. & 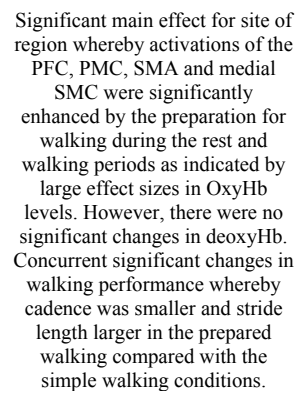 & $\begin{array}{l}\text { No mention of } \\
\text { filtring or } \\
\text { controlling for } \\
\text { movements or } \\
\text { controlling for } \\
\text { in } \\
\text { ambient light. }\end{array}$ & $\begin{array}{l}\text { Preparation of gait shares } \\
\text { similar structures in the } \\
\text { frontal cortex with gait } \\
\text { execution. Preparation for } \\
\text { gait enhanced frontal } \\
\text { activation and influenced } \\
\text { gait performance. }\end{array}$ \\
\hline Takeuchi et al., 2016 & $\begin{array}{l}15 \text { HAA, } 71.7 \pm 3.3 \\
\text { years old, } 5 \text { womenn, } \\
15 \text { healty young } \\
\text { adults, } 25.9 \pm \pm 4.4 \\
\text { years old, } 5 \text { women. }\end{array}$ & $\begin{array}{l}\text { To evaluate the } \\
\text { correlation between PFC } \\
\text { activity and DT cost } \\
\text { during smartphone use } \\
\text { while walking in young } \\
\text { and HOA. }\end{array}$ & $\begin{array}{l}\text { Baseline: overground } \\
\text { walking on a } 2.5 \mathrm{~m} \\
\text { radius pathway at self- } \\
\text { selected speed for } 30 \mathrm{~s} 5 \\
\text { trials). Geit outcomes: } \\
\text { step time and } \\
\text { acceleration magnitude. }\end{array}$ & 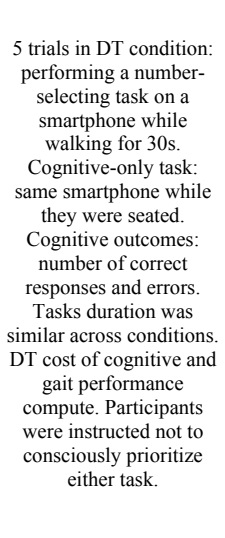 & 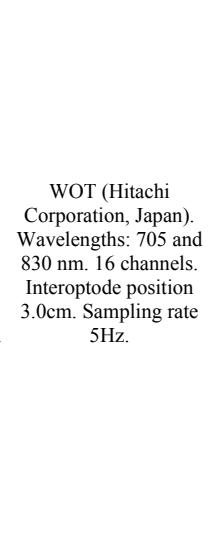 & Охунь & $\begin{array}{l}\text { PFC, } \\
\text { divided in } \\
\text { left, } \\
\text { middle } \\
\text { and right } \\
\text { PFC. }\end{array}$ & 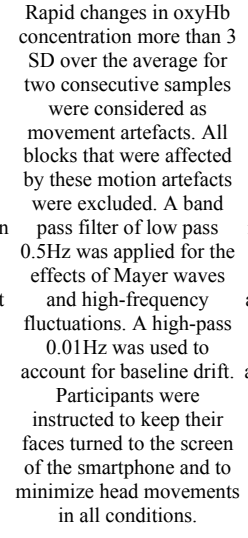 & 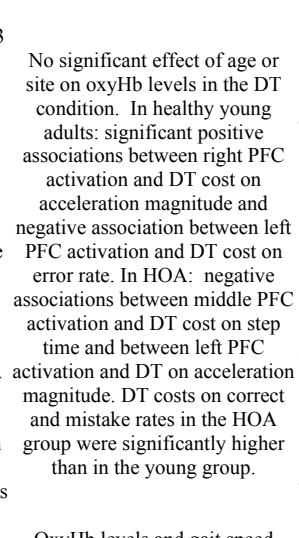 & 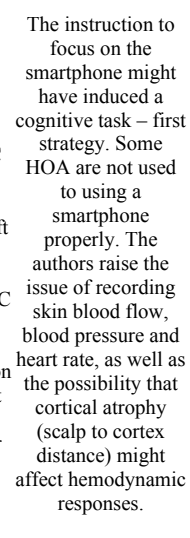 & 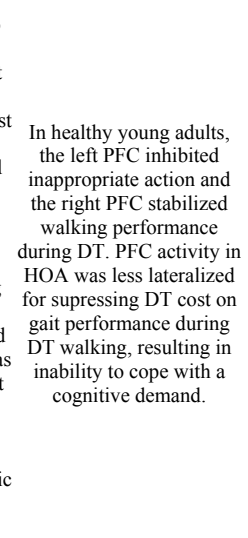 \\
\hline Thumm et al., 2018 & $\begin{array}{l}20 \text { people with } \mathrm{PD}, \\
69.8 \pm 6.5 \text { years old, } \\
10 \text { women. }\end{array}$ & $\begin{array}{l}\text { To investigate whether } \\
\text { during treadmill walking, } \\
\text { PFC activation in people } \\
\text { with PD is lower as } \\
\text { compared to overground } \\
\text { walking. }\end{array}$ & $\begin{array}{l}\text { Five 30s-trials at self- } \\
\text { selected speed in each } \\
\text { condition in the } \\
\text { following fixed order: } \\
\text { (i) overground walk: in } \\
\text { a } 30 \mathrm{~m} \text { corridor; (ii) } \\
\text { treaddill walk. } \\
\text { Baseline: } 20 \text { standing } \\
\text { still before and after } \\
\text { each-trial. Gait } \\
\text { outcomes: gait speed } \\
\text { and stride time. }\end{array}$ & None. & 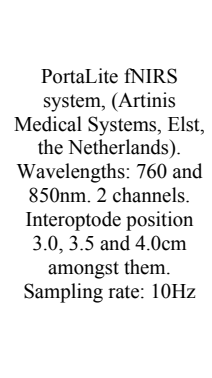 & Охунь. & PFC. & 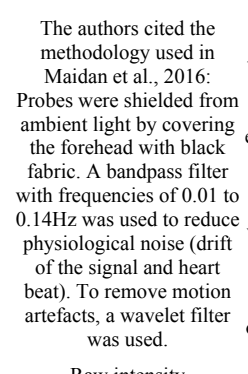 & 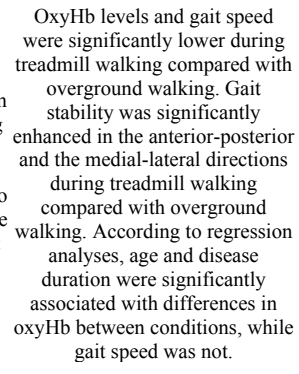 & $\begin{array}{l}\text { The authors } \\
\text { heported that they } \\
\text { did not controf for } \\
\text { superficial } \\
\text { semodynamics or } \\
\text { systemic changes } \\
\text { like blood flow and } \\
\text { nor heart rate or } \\
\text { lower rimb } \\
\text { kinematics in the } \\
\text { two walking } \\
\text { le conditions. }\end{array}$ & $\begin{array}{l}\text { The findings support the } \\
\text { idea that when gait } \\
\text { extermally paced, } \\
\text { activation is reduced in } \\
\text { a people with PD, perhaps } \\
\text { peflecting areduced need } \\
\text { ror compensatory } \\
\text { cognitive mechanisms. }\end{array}$ \\
\hline Verghese et al., 2017 & $\begin{array}{l}166 \mathrm{HOA}, 75.0 \pm \\
6.1 \text { years old, } 85 \% \\
\text { women. }\end{array}$ & $\begin{array}{l}\text { To determinin wheterher } \\
\text { PFC activity during } \\
\text { walking predicts falls in } \\
\text { HOA. }\end{array}$ & 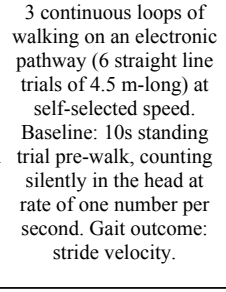 & $\begin{array}{l}\text { Reciting alternate letters } \\
\text { of the alphabet while } \\
\text { walking } 3 \text { continuous } \\
\text { loops as in the single } \\
\text { walking task ( } 6 \text { straight } \\
\text { line trials of } 4.5 \mathrm{~m} \text {-long). } \\
\text { Instruction to pay equal } \\
\text { attention to their walking } \\
\text { and talking. Outcomes: } \\
\text { letter rate and errors per } \\
\text { minute. }\end{array}$ & 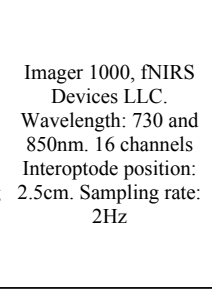 & Охунь. & PFC. & $\begin{array}{l}\text { Raw intensity } \\
\text { measurements at } 730 \text { and } \\
855 \text { nm were low-pass } \\
\text { filtered with a finite } \\
\text { inpulse response filter } \\
\text { with a cut-off frequency of } \\
0.14 \text { Hz. This procedure } \\
\text { was adopted to eliminate } \\
\text { artefacts due to } \\
\text { respiration, heart rate } \\
\text { signals and unwanted high } \\
\text { frequency noise. } \\
\end{array}$ & 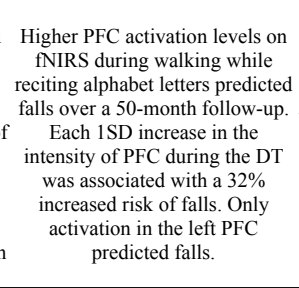 & $\begin{array}{l}\text { Unknown if lightin } \\
\text { conditions were } \\
\text { controlled for }\end{array}$ & $\begin{array}{l}\text { PFC activity levels while } \\
\text { g performing a cognitively } \\
\text { demanding walking } \\
\text { condition predicted falls in } \\
\text { HOA. }\end{array}$ \\
\hline
\end{tabular}




\section{Table 2 (on next page)}

Table 2: Effect of the additional tasks relative to baseline conditions on regional cortical activation in healthy young adults, healthy older adults and clinical groups with balance disorders.

PFC: Prefrontal cortex; preSMA: Pre-supplementary motor area; SMA: Supplementary motor area; S1: Primary sensorimotor cortex; M1: Primary motor cortex; PMC: Premotor cortex; mSMC: Medial sensorimotor cortex; $(+)$ higher activation when performing the additional task; (-) lower activation when performing the additional task; (=) no changes in activation;

Baseline comparison: ${ }^{1}$ standing still; ${ }^{2}$ simple walking; ${ }^{3}$ easier level of the secondary task.

Significant differences only for: ${ }^{4}$ first half of the task; ${ }^{5}$ second half of the task; ${ }^{6}$ obstacle negotiation and wearing a vest with $10 \%$ body weight conditions; ${ }^{7}$ walk vs. visual task; ${ }^{8}$ walk vs alphabet recall; ${ }^{9}$ overground walking; ${ }^{10}$ walking and counting backwards task. ${ }^{a}$ Ataxia; ${ }^{\mathrm{b}}$ Stroke; 'Parkinson's disease; ' ${ }^{\mathrm{d}}$ Obesity; ${ }^{\mathrm{e}}$ Multiple Sclerosis; ${ }^{\mathrm{f}}$ Mild Cognitive Impairment; ${ }^{9}$ Neurological gait. 


\begin{tabular}{|c|c|c|c|}
\hline & Healthy young adults & Healthy older adults & $\begin{array}{c}\text { Clinical groups with balance } \\
\text { disorders }\end{array}$ \\
\hline \multicolumn{4}{|l|}{ Simple walking } \\
\hline Caliandro et al., $\mathbf{2 0 1 5}^{1}$ & & $\operatorname{PFC}(=)$ & $\operatorname{PFC}(+)^{\mathrm{a}}$ \\
\hline Lin; Lin, 2016 $^{1}$ & $\operatorname{PFC}(+)$ & & \\
\hline Hawkins et al., $2018^{1}$ & $\operatorname{PFC~(-)~}$ & $\operatorname{PFC}(+)^{4}, \operatorname{PFC}(=)^{5}$ & $\operatorname{PFC}(+)^{4, b}, \operatorname{PFC}(=)^{5, b}$ \\
\hline Thumm et al., $2018^{1}$ & & & $\operatorname{PFC}(+)^{\mathrm{c}}$ \\
\hline \multicolumn{4}{|l|}{ Fast walking } \\
\hline Harada et al., $2009^{1}$ & & $\begin{array}{l}\text { left PFC }(+), \text { right PFC }(=), \\
\text { preSMA }(=), \text { SMA }(+), \text { mSMC } \\
(=)\end{array}$ & \\
\hline Eggenberger et al., $2016^{2}$ & & $\operatorname{PFC}(=)$ & \\
\hline Suzuki et al., 2004² & $\operatorname{PFC}(+), \operatorname{PMC}(=), \operatorname{SMC~}(=)$ & & \\
\hline \multicolumn{4}{|l|}{ Motor task } \\
\hline Chen et al., 2017² & & $\operatorname{PFC}(+)^{6}$ & \\
\hline Clark et al., 2014b ${ }^{2}$ & & $\operatorname{PFC}(+)^{6}$ & \\
\hline Koenraadt et al., $2014^{2}$ & $\begin{array}{l}\text { PFC }(-)^{4}, \text { PFC }(=)^{5}, \text { preSMA } \\
(=), \text { SMA }(=), \text { S1 }(=), \text { M1 }(=)\end{array}$ & & \\
\hline Lin; Lin, 2016 $^{1}$ & PFC (-) & & \\
\hline Lu et al., $2015^{2}$ & left PFC $(+)$, SMA $(+)$ & & \\
\hline Maidan et al., $2016^{2}$ & & $\operatorname{PFC}(=)$ & $\operatorname{PFC}(+)^{\mathrm{c}}$ \\
\hline Maidan et al., $2018^{2}$ & $\operatorname{PFC}(+)$ & & \\
\hline Mirelman et al., $2017^{2}$ & $\mathrm{PFC}(+)$ & $\operatorname{PFC}(+)$ & \\
\hline Osofundiya et al., 2016 ${ }^{1,2}$ & & $\operatorname{PFC~}(+)$ & $\operatorname{PFC}(+)^{\mathrm{d}}$ \\
\hline Hawkins et al., $2018^{1}$ & PFC (-) & $\operatorname{PFC}(=)$ & $\operatorname{PFC}(+)^{\mathrm{b}}$ \\
\hline \multicolumn{4}{|l|}{ Somatosensory task } \\
\hline Chaparro et al., 20171,3 & & $\operatorname{PFC}(+)$ & $\operatorname{PFC}(+)^{\mathrm{e}}$ \\
\hline Clark et al., 2014a ${ }^{2}$ & & PFC (-) & \\
\hline Clark et al., 2014b ${ }^{2}$ & & $\operatorname{PFC}(=)$ & \\
\hline \multicolumn{4}{|l|}{ Cognitive task } \\
\hline Al-Yahya et al., $2016^{2}$ & & $\operatorname{PFC}(=)$ & $\operatorname{PFC}(+)^{\mathrm{b}}$ \\
\hline Beurskens et al., 2014² & $\operatorname{PFC}(=)$ & $\operatorname{PFC}(-)^{7}, \operatorname{PFC}(=)^{8}$ & \\
\hline Chaparro et al., $2017^{1,3}$ & & $\operatorname{PFC~}(+)$ & $\operatorname{PFC}(+)^{\mathrm{e}}$ \\
\hline Chen et al., 2017 & & $\operatorname{PFC}(+)$ & \\
\hline Clark et al., $2014 \mathrm{a}^{2}$ & & $\operatorname{PFC}(+)^{9}$ & \\
\hline Clark et al., 2014b² & & $\operatorname{PFC~}(+)$ & \\
\hline Doi et al., $2013^{2}$ & & & $\operatorname{PFC}(+)^{\mathrm{f}}$ \\
\hline Hernandez et al., $2016^{2}$ & & $\operatorname{PFC}(+)$ & $\operatorname{PFC}(+)^{\mathrm{e}}$ \\
\hline Hill et al., $2013^{3}$ & left PFC (+) & & \\
\hline Hawkins et al., $\mathbf{2 0 1 8}^{1}$ & $\operatorname{PFC}(=)$ & $\operatorname{PFC}(+)^{4}, \operatorname{PFC}(=)^{5}$ & $\operatorname{PFC}(+)^{\mathrm{b}}$ \\
\hline Holtzer et al., 2011² & $\operatorname{PFC}(+)$ & $\operatorname{PFC}(+)$ & \\
\hline Holtzer et al., $2015^{2}$ & & $\operatorname{PFC}(+)$ & \\
\hline Holtzer et al., 2016 ${ }^{2}$ & & $\operatorname{PFC}(+)$ & $\operatorname{PFC}(+)^{\mathrm{g}}$ \\
\hline Holtzer et al., 2017 $\mathbf{a}^{2}$ & & $\operatorname{PFC}(+)$ & \\
\hline Holtzer et al., 2017b ${ }^{2}$ & & $\operatorname{PFC}(+)$ & \\
\hline Lin; Lin, 2016 ${ }^{1}$ & PFC (-) & & \\
\hline Lu et al., $2015^{2}$ & PFC (+), PMC (+), SMA (+) & & \\
\hline Lucas et al., $2018^{2}$ & & $\operatorname{PFC}(+)$ & \\
\hline Maidan et al., 2016 2 & & $\operatorname{PFC}(+)$ & $\operatorname{PFC}(=)^{\mathrm{c}}$ \\
\hline Meester et al., 2014² & right $\mathrm{PFC}(+)$ & & \\
\hline Mirelman et al., $\mathbf{2 0 1 4}^{2}$ & $\operatorname{PFC}(+)^{10}$ & & \\
\hline Mirelman et al., $2017^{2}$ & $\operatorname{PFC}(+)$ & $\operatorname{PFC}(+)$ & \\
\hline Mori et al., 2018 ${ }^{1,2}$ & & $\operatorname{PFC}(=)$ & $\operatorname{PFC}(=)^{b}$ \\
\hline
\end{tabular}


Nieuwholf et al., 2016 ${ }^{1}$

Osofundiya et al., 2016 ${ }^{1,2}$

Takeuchi et al., 2016 ${ }^{1}$

Verghese et al., 2017 1

\begin{tabular}{|c|c|c|}
\hline & & $\operatorname{PFC}(+)^{c}$ \\
\hline & $\operatorname{PFC}(+)$ & $\operatorname{PFC}(+)$ \\
\hline \multirow[t]{2}{*}{$\operatorname{PFC}(=)$} & $\operatorname{PFC}(=)$ & \\
\hline & $\operatorname{PFC}(+)$ & \\
\hline
\end{tabular}

1 


\section{Table 3 (on next page)}

Table 3: Methodological reporting criteria ratings for the included studies. 


\begin{tabular}{|c|c|c|c|c|c|c|c|c|}
\hline Study & $\begin{array}{l}\text { Equipment } \\
\text { details } \\
\text { provided }\end{array}$ & $\begin{array}{l}\text { Movement } \\
\text { artefacts } \\
\text { considered }\end{array}$ & $\begin{array}{l}\text { Optode } \\
\text { placement } \\
\text { specified }\end{array}$ & $\begin{array}{c}\text { External } \\
\text { light } \\
\text { confounding } \\
\text { effect } \\
\text { considered } \\
\end{array}$ & $\begin{array}{c}\text { Heart changes } \\
\text { confounding } \\
\text { effect } \\
\text { considered }\end{array}$ & $\begin{array}{c}\text { Sample size } \\
\text { (n>10 per } \\
\text { group) }\end{array}$ & Score & Quality criteria \\
\hline Beurskens et al., 2014 & 1 & 1 & 1 & 1 & 1 & 1 & 6 & High quality \\
\hline Caliandro et al., 2015 & 1 & 1 & 1 & 0 & 1 & 1 & 5 & High quality \\
\hline Chaparro et al., 2017 & 1 & 0 & 0 & 0 & 1 & 1 & 3 & Medium quality \\
\hline Clark et al., 2014a & 0 & 0 & 0 & 0 & 0 & 1 & 1 & Low quality \\
\hline Clark et al., 2014b & 0 & 0 & 0 & 0 & 0 & 1 & 1 & Low quality \\
\hline Doi et al., 2013 & 1 & 1 & 1 & 0 & 0 & 1 & 4 & Medium quality \\
\hline Eggenberger et al., 2016 & 1 & 1 & 1 & 0 & 1 & 1 & 5 & High quality \\
\hline Harada et al., 2009 & 1 & 0 & 1 & 0 & 1 & 1 & 5 & High quality \\
\hline Hawkins et al.,2018 & 1 & 1 & 0 & 0 & 0 & 0 & 2 & Low quality \\
\hline Holtzer et al., 2016 & 1 & 1 & 1 & 0 & 1 & 1 & 5 & High quality \\
\hline Holtzer et al., 2017a & 1 & 1 & 0 & 0 & 1 & 1 & 4 & Medium quality \\
\hline Holtzer et al., 2017b & 1 & 1 & 0 & 0 & 1 & 1 & 4 & Medium quality \\
\hline Koenraadt et al., 2014 & 1 & 1 & 1 & 0 & 1 & 1 & 5 & High quality \\
\hline Lin \& Lin, 2016 & 1 & 0 & 1 & 0 & 0 & 1 & 3 & Medium quality \\
\hline Lu et al., 2015 & 1 & 1 & 1 & 0 & 1 & 1 & 5 & High quality \\
\hline Lucas et al., 2018 & 1 & 1 & 1 & 0 & 1 & 1 & 5 & High quality \\
\hline Maidan et al., 2016 & 1 & 1 & 0 & 1 & 1 & 1 & 5 & High quality \\
\hline Maidan et al., 2018 & 1 & 1 & 1 & 1 & 1 & 1 & 6 & High quality \\
\hline Meester et al., 2014 & 1 & 1 & 1 & 0 & 1 & 1 & 5 & High quality \\
\hline Mirelman et al., 2014 & 1 & 0 & 1 & 0 & 0 & 1 & 3 & Medium quality \\
\hline
\end{tabular}


Osofundiya et al., 2016

Suzuki et al., 2008

Suzuki et al., 2004

Takeuchi et al., 2016

Thumm et al., 2018

Verghese et al., 2017

$\begin{array}{ll}0 & 1 \\ 0 & 0 \\ 0 & 0 \\ 0 & 1 \\ 0 & 1 \\ 1 & 1\end{array}$

1
0
0
0
0
0

1
0
1
0
0
1

$\begin{array}{ll}1 & 4 \\ 0 & 1 \\ 0 & 2 \\ 1 & 3 \\ 1 & 3 \\ 1 & 5\end{array}$

Medium quality

Low quality

Low quality

Medium quality

Medium quality

High quality 
Table 4 (on next page)

Table 4: Prefrontal cortical activation in relation to methodological reporting scale. 


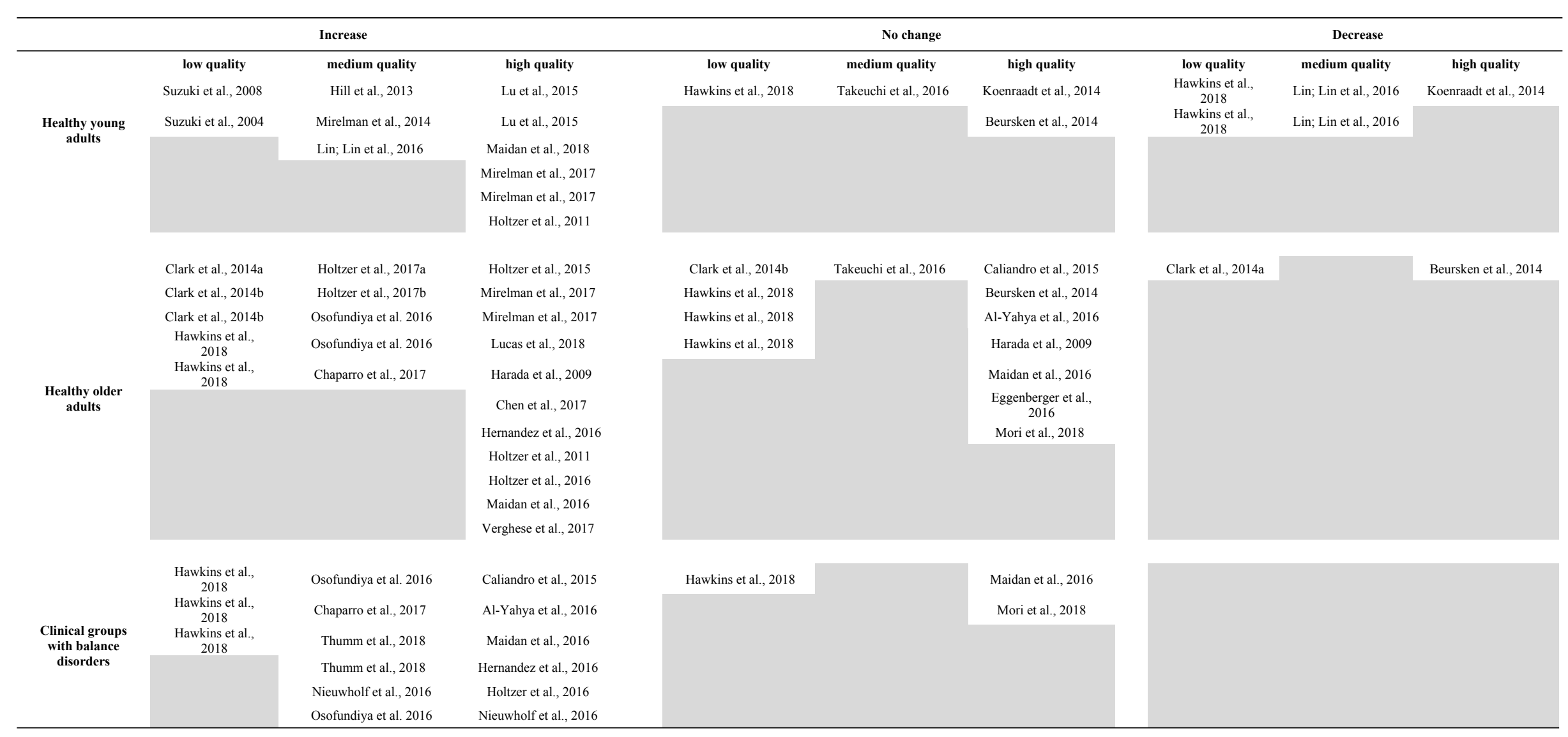




\section{Table 5 (on next page)}

Table 5: Prefrontal cortical activation in relation to complex walking tasks. 


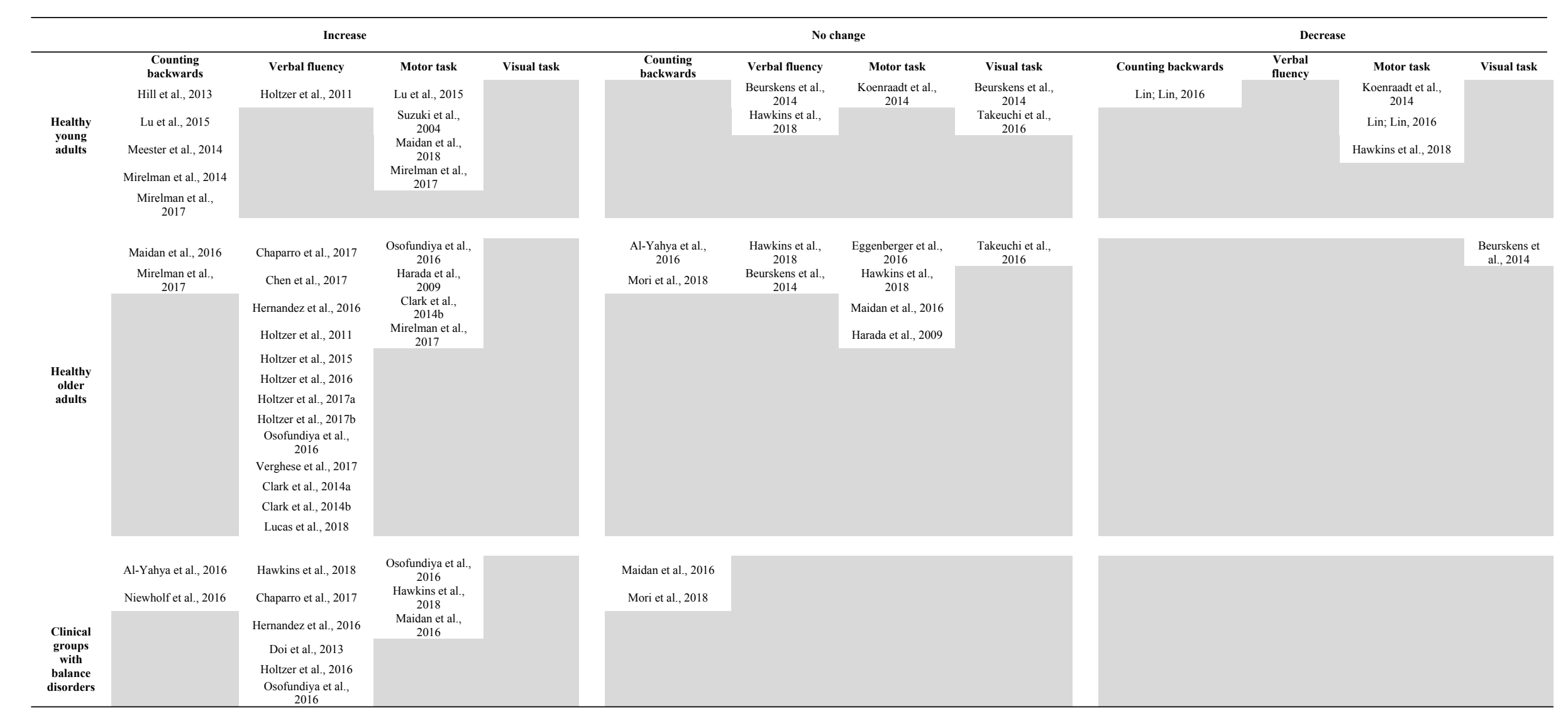




\section{Table 6(on next page)}

Table 6: Effect of the additional tasks on gait outcomes compared to simple walking in healthy young adults, healthy older adults and clinical groups with balance disorders.

$(+)$ increase of spatiotemporal parameter when performing the additional task during walking; (-) decrease of spatiotemporal parameter when performing the additional task; (=) no changes in spatiotemporal parameter. Significant differences only for: ${ }^{1}$ obstacle negotiation; ${ }^{2}$ treadmill walking; ${ }^{3}$ overground walking; ${ }^{4}$ walking + subtracting by $7 \mathrm{~s} ;{ }^{5}$ walking while reciting digit spans. 


\begin{tabular}{|c|c|c|c|}
\hline & Healthy young adults & Healthy older adults & Clinical groups with balance disorders \\
\hline \multicolumn{4}{|c|}{ Motor secondary task } \\
\hline Chen et al., 2017 & & Gait speed (-) & \\
\hline Clark et al., 2014b & & $\begin{array}{c}\text { Gait speed }(-), \\
\text { step length variability }(+)^{1}\end{array}$ & \\
\hline Hawkins et al., 2018 & Gait speed (-) & Gait speed (-) & Gait speed (-) \\
\hline Koenraadt et al., 2014 & Step time variability $(+)$ & & \\
\hline Lin; Lin, 2016 & Gait speed (-), step length (-) & & \\
\hline Lu et al., 2015 & $\begin{array}{c}\text { Gait speed (-), cadence }(=), \\
\text { stride length }(-), \text { gait variability }(=)\end{array}$ & & \\
\hline Mirelman et al., 2017 & $\begin{array}{c}\text { Gait speed }(-), \text { stride length }(+), \\
\text { gait variability }(+)\end{array}$ & $\begin{array}{l}\text { Gait speed }(=) \text {,stride length }(+), \\
\text { gait variability }(+)\end{array}$ & \\
\hline Osofundiya et al., 2016 & & Gait speed (-) & Gait speed (-) \\
\hline \multicolumn{4}{|l|}{ Somatossensory task } \\
\hline Clark et al., 2014a & & $\begin{array}{l}\text { step length }(=)^{2} \text {, gait speed }(=)^{2}, \\
\text { step length }(-)^{3} \text {, gait speed }(-)^{3}, \\
\text { step length variability }(=)^{2,3}\end{array}$ & \\
\hline Clark et al., 2014b & & gait speed $(=)$, step length variability $(=)$ & \\
\hline Chaparro et al., 2017 & & Stride length (-) & Stride length (-) \\
\hline \multicolumn{4}{|l|}{ Cognitive secondary task } \\
\hline Al-Yahya et al., 2016 & & Stride length (+), cadence (-) & Stride length $(+)$, cadence (-) \\
\hline Beurskens et al., 2014 & Step length (-) & Step length (-) & \\
\hline Chaparro et al., 2017 & & Stride length $(=)$ & Stride length $(=)$ \\
\hline Clark et al., 2014a & & $\begin{array}{c}\text { step length }(-)^{2,3}, \\
\text { step length variability }(+)^{2,3} \\
\text { gait speed }(=)^{2} \text {, gait speed }(-)^{3}\end{array}$ & \\
\hline Clark et al., 2014b & & Gait speed (-), step length variability $(=)$ & \\
\hline Doi et al., 2013 & & & Gait speed (-) \\
\hline Hernandez et al., 2016 & & Gait speed (-) & Gait speed (-) \\
\hline Hill et al., 2013 & Gait speed (-) & & \\
\hline Holtzer et al., 2011 & Gait speed (-) & Gait speed (-) & \\
\hline Holtzer et al., 2015 & & Stride length (-), gait speed (-) & \\
\hline Holtzer et al., 2016 & & Gait speed (-) & Gait speed (-) \\
\hline Holtzer et al., 2017a & & Gait speed (-) & \\
\hline Holtzer et al., 2017b & & Gait speed (-) & \\
\hline Lin; Lin, 2016 & Gait speed (-), step length (-) & & \\
\hline Lu et al., 2015 & $\begin{array}{l}\text { Gait speed (-), cadence }(-), \\
\text { stride length }(-) \text {, gait variability }(=)\end{array}$ & & \\
\hline Lucas et al., 2018 & & Gait speed (-) & \\
\hline Meester et al., 2014 & Step time variability $(=)$ & & \\
\hline Mirelman et al., 2014 & Stride length $(-)^{4}$, gait speed (-) & & \\
\hline Mirelman et al., 2017 & $\begin{array}{c}\text { Gait speed }(-), \text { stride length }(=) \text {, gait } \\
\text { variability }(=)\end{array}$ & $\begin{array}{c}\text { Gait speed }(=) \text {,stride length }(=) \text {, gait } \\
\text { variability }(=)\end{array}$ & \\
\hline Nieuwholf et al., 2016 & & & $\begin{array}{c}\text { Stride length }(-)(\mathrm{e}) \\
\text { Stride length variability }(-)^{5}\end{array}$ \\
\hline Osofundiya et al., 2016 & & Gait speed (-) & Gait speed (-) \\
\hline Verghese et al., 2017 & & Gait speed (-) & \\
\hline
\end{tabular}




\section{Table 7 (on next page)}

Table 7: Prefrontal cortical activation pattern differences between healthy young and older adults and between clinical groups with balance disorders and healthy peers.

DT: Dual-task; SW: Simple walking; OBS: Obstacle negotiation; a: first half of the task; b: second half of the task; PB: Partial body support; PS: Precision stepping; (+) higher activation when performing the additional task; (-) lower activation when performing the additional task; (=) no change in activation. 


\section{PFC activity}

\section{Healthy older vs. healthy young adults}

Beurskens et al., 2014

Holtzer et al., 2011

Takeuchi et al., 2016

Hawkins et al., 2018

Mirelman et al., 2017

Clinical group with balance disorders vs. healthy peers

Al-Yahya et al., 2016 (Stroke)

Chaparro et al., 2017 (Multiple Sclerosis)

Hawkins et al., 2018 (Stroke)

Hernandez et al., 2016 (Multiple Sclerosis)

Holtzer et al., 2016 (Neurological gait)

Maidan et al., 2016 (Parkinson's disease)

Mori et al., 2018 (Stroke)

Osofundiya et al., 2016 (Obesity)
DT (=)

DT (-)

DT (=)

SW (+)/ OBS a $(+) /$ OBS b (=)/ DT (=)

SW (+)/ OBS (+)/ DT (+) 